\title{
Tensor-Centric Warfare IV: Kähler Dynamics of Battlefields
}

\author{
Vladimir Ivancevic ${ }^{1}$, Darryn Reid ${ }^{1}$, Peyam Pourbeik ${ }^{2}$ \\ ${ }^{1}$ Joint and Operations Analysis Division, Defence Science \& Technology Group, Adelaide, Australia \\ ${ }^{2}$ Cyber and Electronic Warfare Division, Defence Science \& Technology Group, Adelaide, Australia \\ Email: Vladimir.Ivancvic@dsto.defence.gov.au, Darryn.Reid@dsto.defence.gov.au, Peyam.Pourbeik@dsto.defence.gov.au
}

How to cite this paper: Ivancevic, V., Reid, D. and Pourbeik, P. (2018) Tensor-Centric Warfare IV: Kähler Dynamics of Battlefields. Intelligent Control and Automation, 9, 123-146. https://doi.org/10.4236/ica.2018.94010

Received: September 28, 2018

Accepted: November 12, 2018

Published: November 15, 2018

Copyright (c) 2018 by authors and Scientific Research Publishing Inc. This work is licensed under the Creative Commons Attribution International License (CC BY 4.0).

http://creativecommons.org/licenses/by/4.0/

\section{c) (i) Open Access}

\begin{abstract}
This paper presents the complex dynamics synthesis of the combat dynamics series called tensor-centric warfare (TCW; for the first three parts of the series, see [1] [2] [3]), which includes tensor generalization of classical Lanchester-type combat equations, entropic Lie-dragging and commutators for modeling warfare uncertainty and symmetry, and various delta-strikes and missiles (both deterministic and random). The present paper gives a unique synthesis of the Red vs. Blue vectorfields into a single complex battle-vectorfield, using dynamics on Kähler manifolds as a rigorous framework for extending the TCW concept. The global Kähler dynamics framework, with its rigorous underpinning called the Kähler-Ricci flow, provides not only a new insight into the "geometry of warfare", but also into the "physics of warfare", in terms of Lagrangian and Hamiltonian structures of the battlefields. It also provides a convenient and efficient computational framework for entropic wargaming.
\end{abstract}

\section{Keywords}

Tensor-Centric Warfare, Kähler Geometry, Complex Battle-Vectorfield, Lagrangian and Hamiltonian Battlefields, Kähler-Ricci Flow, Entropic Computational Wargaming

\section{Introduction}

In the series of papers called the tensor-centric warfare (TCW; see [1] [2] [3]) we have developed a tensor architecture for general Red-Blue combat dynamics. The TCW framework starts by providing tensorial generalization of the Lanchester-type combat equations [1], and then includes entropic Lie-dragging (for modeling warfare uncertainty) and commutators (for analysis of warfare 
symmetry) [2], as well as various kinds of delta-strikes and missiles [3]. The whole TCW architecture is defined by the following pair of Red-and-Blue tensorial dynamical systems (formally, the pair of Red-Blue vectorfields):

Red : $\overbrace{\partial_{t} R^{a}}^{\text {Red.vecfield }}=\overbrace{k A_{b}^{a} B^{b}}^{\text {lin.Lanchaster }}+\overbrace{k_{b} F_{c d}^{a b} R^{c} B^{d}}^{\text {quad.Lanchaster }}+\overbrace{R^{b} \mathcal{L}_{R} \mathcal{N}_{b}^{a}}^{\text {Lie.dragging }}+\overbrace{\left[R^{a}, B^{a}\right]}^{\text {war.symmetry }}+\overbrace{\delta R^{a}(\mathrm{H}-\mathrm{L})}^{\text {delta.strikes }}$,

Blue : $\overbrace{\partial_{t} B^{a}}^{\text {Blue.vecfield }}=\overbrace{\kappa C_{b}^{a} R^{b}}^{\text {lin.Lanchaster }}+\overbrace{\kappa_{b} G_{c d}^{a b} R^{c} B^{d}}^{\text {quad.Lanchaster }}+\overbrace{B^{b} \mathcal{L}_{B} \mathcal{N}_{b}^{a}}^{\text {Lie.dragging }}+\overbrace{\left[B^{a}, R^{a}\right]}^{\text {war.symmetry }}+\overbrace{\delta B^{a}(\mathrm{H}-\mathrm{L})}^{\text {delta.strikes }}$.

In Equations (1) the Red and Blue Hamilton-Langevin delta strikes, $\delta R^{a}(\mathrm{H}-\mathrm{L})$ and $\delta B^{a}(\mathrm{H}-\mathrm{L})$, partially derived from the Ising-type battle Hamiltonian: $H=-J_{a b} R^{a} B^{b}$, with the connection tensor:

$J_{a b}=A_{b}^{a} C_{a}^{b} \eta_{a b}$ (weighted by the random noise; see [3] for details), read:

$$
\begin{aligned}
& \delta R^{a}(\mathrm{H}-\mathrm{L})=\overbrace{\sum_{j=1}^{N} \alpha_{j}^{a} \delta\left(t-\tau_{j}^{R}\right)}^{\text {disc.spectrum }}+\overbrace{\int_{t_{0}}^{t_{1}} \alpha^{a}(t) \delta\left(t-\tau_{R}\right) \mathrm{d} t}^{\text {cont.spectrum }}+\overbrace{\alpha^{a} \sum_{j=1}^{N} \delta\left(t-\tau_{j}^{R}\right)\left( \pm \rho^{R}\right)}^{\text {bidirect.rnd }} \\
& +\overbrace{\left[R^{a}, B^{a}\right] \frac{\partial H}{\partial B^{b}} R^{b}}^{\text {RedHam.vecfield }}-\overbrace{\gamma_{b}^{a} R^{b}}^{\text {self.dissipat }}-\overbrace{\gamma^{a b} \frac{\partial H}{\partial B^{b}}}^{\text {oppon.dissipat }}+\overbrace{f_{\text {rnd }}^{a}(t)}^{\text {rnd.force }}, \\
& \delta B^{a}(\mathrm{H}-\mathrm{L})=\overbrace{\sum_{j=1}^{M} \beta_{j}^{a} \delta\left(t-\tau_{j}^{B}\right)}^{\text {disc.spectrum }}+\overbrace{\int_{t_{0}}^{t_{1}} \beta^{a}(t) \delta\left(t-\tau_{B}\right) \mathrm{d} t}^{\text {cont.spectrum }}+\overbrace{\beta^{a} \sum_{j=1}^{M} \delta\left(t-\tau_{j}^{B}\right)\left( \pm \rho^{B}\right)}^{\text {bidirect.rnd }} \\
& +\overbrace{\left[B^{a}, R^{a}\right] \frac{\partial H}{\partial R^{b}} B^{b}}^{\text {BlueHam.vecfield }}-\overbrace{\chi_{b}^{a} B^{b}}^{\text {self.dissipat }}-\overbrace{\chi^{a b} \frac{\partial H}{\partial R^{b}}}^{\text {oppon.dissipat }}+\overbrace{g_{\text {rnd }}^{a}(t)}^{\text {rnd.force }} .
\end{aligned}
$$

In the Red-Blue Equations (1)-(2), $\partial_{t} \equiv \partial / \partial t$ and the Red and Blue forces are defined as vectors $R^{a}=R^{a}(x, t) \in M_{\mathrm{Red}}$ and $B^{a}=B^{a}(q, t) \in M_{\text {Blue }}$, defined on their respective configuration $n$-manifolds $M_{\text {Red }}$ (with local coordinates $\left\{x^{a}\right\}$, for $a=1, \cdots, n)$ and $M_{\text {Blue }}$ (with local coordinates $\left\{q^{a}\right\}$ ). The Red and Blue vectorfields, $\partial_{t} R^{a}$ and $\partial_{t} B^{a}$, include the following terms (placed on the right-hand side of Equations (1)):

- Linear Lanchester-type terms, $k A_{b}^{a} B^{b} \in M_{\text {Red }}$ and $\kappa C_{b}^{a} R^{b} \in M_{\text {Blue }}$, with combat tensors $A_{b}^{a}$ and $C_{b}^{a}$ defined via bipartite and tripartite adjacency matrices, respectively defining Red and Blue aircraft formations (according to the aircraft-combat scenario from [4] and [1]);

- Quadratic Lanchester-type terms, $k_{b} F_{c d}^{a b} B^{c} R^{d} \in M_{\mathrm{Red}}$ and $\kappa_{b} G_{c d}^{a b} B^{c} R^{d} \in M_{\text {Blue }}$, with the 4 th-order tensors $F_{c d}^{a b}$ and $G_{c d}^{a b}$ representing strategic, tactical and operational capabilities of the Red and Blue forces (see $[1])$;

- Entropic Lie-dragging of the opposite side terms, $R^{b} \mathfrak{L}_{R} \mathfrak{N}_{b}^{a} \in M_{\text {Red }}$ and $B^{b} \mathfrak{L}_{B} \mathcal{N}_{b}^{a} \in M_{\text {Blue }}$, where $\mathfrak{N}_{b}^{a}=C_{b}^{a}+G_{b c}^{c a}$ and $\mathcal{N}_{b}^{a}=A_{b}^{a}+F_{b c}^{c a}$. In case of resistance, the Lie derivatives are positive, $\left|\mathfrak{L}_{R} \mathfrak{N}_{b}^{a}\right|>0$ and $\left|\mathfrak{L}_{B} \mathcal{N}_{b}^{a}\right|>0$, so that the non-equilibrium battlefield entropy grows, $\partial_{t} S>0$; in case of non-resistance, the Lie derivatives vanish, $\left|\mathfrak{L}_{R} \mathfrak{N}_{b}^{a}\right|=0$ and $\left|\mathfrak{L}_{B} \mathcal{N}_{b}^{a}\right|=0$, so that the battlefield entropy is conserved, $\partial_{t} S=0$ (see [2]);

- Entropic Red-Blue commutators, $\left|\left[R^{a}, B^{a}\right]\right| \geq 0 \in M_{\mathrm{Red}}$ and 
$\left|\left[B^{a}, R^{a}\right]\right| \geq 0 \in M_{\text {Blue }}$, for modeling warfare symmetry (see [2]);

- Hamilton-Langevin delta strikes, $\delta R^{a}(\mathrm{H}-\mathrm{L})$ and $\delta B^{a}(\mathrm{H}-\mathrm{L})$, on both sides, including discrete striking spectra (slow-fire missiles) and continuous striking spectra (rapid-fire missiles), as well as bidirectional random strikes, Hamiltonian vectorfields, self-dissipation, opponent-caused dissipation and non-delta random forces (see [3]).

The first three models of the TCW series have been developed on the Red and Blue configuration manifolds, $M_{\text {Red }}$ and $M_{\text {Blue }}$, intentionally without specifying any geometric structures on these manifolds. In the present paper, we use the most sophisticated geometric structure of Kähler manifolds, which allows development of both Lagrangian and Hamiltonian formalisms on it. Here we summarize and reformulate the two Red-and-Blue dynamical systems (1)-(2) in the form of a unique Kähler dynamical system, together with its specific geometrical underpinning called the Kähler-Ricci flow. This sophisticated geometric framework gives a new insight into deep mathematical and physical structures of battlefields and also provides a convenient computational wargaming framework.

\section{Kähler Dynamics of Battlefields}

The concept of Kähler dynamics, or tensor dynamics on Kähler manifolds (see Appendix 1 for a technical exposition), has been formally developed in [5], based on our previous work on self-organization entropy [6], controllable complexity [7] and autonomy of cyber-physical-cognitive systems [8].

Briefly, the Kähler dynamics is defined by the complex-valued vectorfield:

$$
\partial_{t} V^{a}\left(z^{a}, t\right)=\partial_{t} R^{a}\left(x^{a}, t\right)+\mathrm{i} \partial_{t} B^{a}\left(q^{a}, t\right),
$$

which flows along the Kähler battle-manifold $\mathcal{K}$ defined by the complexified sum (i.e., the sum with the imaginary unit: $i^{2}=1$ ):

$$
\overbrace{\mathcal{K}}^{\partial_{t} V^{a}\left(z^{a}, t\right)}=\overbrace{T M_{\text {Red }}}^{\partial_{t} R^{a}\left(x^{a}, t\right)}+\overbrace{\mathrm{i} T^{*} M_{\text {Blue }}}^{\partial_{t} B^{a}\left(q^{a}, t\right)},
$$

where $T M_{\text {Red }}$ is the tangent bundle (or, velocity phase-space) of the Red forces with Riemannian structure $g_{R}$ and natural Lagrangian dynamics (derived from the Lagrangian energy function $L$ ), and $T^{*} M_{\text {Blue }}$ is the cotangent bundle (or, momentum phase-space) of the Blue forces with symplectic structure $\omega_{S}$ and natural Hamiltonian dynamics (derived from the Hamiltonian energy function H).

More specifically, a Kähler manifold, $\mathcal{K} \equiv(\mathcal{K}, g) \equiv(\mathcal{K}, \omega)$, represents a Hermitian manifold of complex dimension $2 n$ (or, real dimension $4 n$ ), defined by the Hermitian metric form: $g=g_{R}+\mathrm{i} \omega_{S}$, where $g_{R}=g_{a b} d x^{a} d x^{b} \in T M_{\text {Red }}$ is the Riemannian metric on the $T M_{\text {Red }}$ tangent bundle and $\omega_{S}=d p_{a} \wedge d q^{a} \in T^{*} M_{\text {Blue }}$ is the symplectic form on the $T^{*} M_{\text {Blue }}$ cotangent bundle. In our case of the two-party battlefield with the Red forces defined by the (real) configuration n-manifold $M_{\text {Red }}$ (with its tangent bundle $T M_{\text {Red }}=\sqcup_{x \in M_{\text {Red }}} T_{x} M_{\text {Red }}$ which is 
the Riemannian $2 n$-manifold) and the Blue forces defined by the configuration n-manifold $M_{\text {Blue }}$ (with its cotangent bundle $T^{*} M_{\text {Blue }}=\sqcup_{q \in M_{\text {Blue }}} T_{q}^{*} M_{\text {Blue }}$ which is the symplectic $2 n$-manifold), ${ }^{1}$ our Kähler battle-manifold $\mathcal{K}$ is defined as the complexified sum:

$$
\begin{aligned}
& (\mathcal{K}, g)=T M_{\text {Red }}+\mathrm{i} T^{*} M_{\text {Blue }} \text { with the Hermitian metric form } g . \\
& g=g_{R}+\mathrm{i} \omega_{S}, \text { where } g_{R}=g_{a b} d x^{a} d x^{b} \in T M_{\text {Red }}, \omega_{S}=d p_{a} \wedge d q^{a} \in T^{*} M_{\text {Blue }},
\end{aligned}
$$
with the local coordinates on the component bundles, $\left(x^{a}, \dot{x}^{a}\right) \in T M_{\operatorname{Red}}$ and $\left(q^{a}, p_{a}\right) \in T^{*} M_{\text {Blue }}$. For further technical details on Kähler manifolds, see Appendix 1.

The unique battlefield dynamics defined by the battle-vectorfield, $\partial_{t} V^{a}\left(z^{a}, t\right)$, has several advantages over the real-valued Red-Blue Equations (1)-(2):

- $\partial_{t} V^{a}\left(z^{a}, t\right)$ is mathematically more consistent than the pair $\left[\partial_{t} R^{a}\left(x^{a}, t\right), \partial_{t} B^{a}\left(q^{a}, t\right)\right]$, since dynamics in the complex plane $\mathbb{C}$ includes dynamics in the real plane $\mathbb{R}^{2}$ but reveals much reacher structure (including polar form, Euler relation, conjugation, etc.);

- $\partial_{t} V^{a}\left(z^{a}, t\right)$ has a rigorous geometric underpinning called the Kähler-Ricci flow;

- $\partial_{t} V^{a}\left(z^{a}, t\right)$ gives a new insight into the physics of warfare in terms of its natural/embedded Lagrangian and Hamiltonian dynamics, and

- $\partial_{t} V^{a}\left(z^{a}, t\right)$ has a straightforward implementation in the computational wargame called the Entropy Battle.

Deep mathematical and physical aspects of this new concept are briefly defined in the next two subsections, based on the rigorous technical exposition given in Appendix 1.

\subsection{Geometry of Warfare: Kähler Battle-Vectorfield}

Our Kähler dynamics of the battlefield is defined as a complex-valued $n \mathrm{D}$ vectorfield $\partial_{t} V^{a}\left(z^{a}, t\right)$, called the Kähler battle-vectorfield, flowing along the Kähler battle-manifold $\mathcal{K}$ and defined in the following two steps. Firstly, the above two real-valued Red-and-Blue vectorfields (1) can be rewritten in terms of the real and imaginary components of a single complex-valued vectorfield, defined on $\mathcal{K}$ as:

$$
\begin{aligned}
\operatorname{Red}: \partial_{t} \operatorname{Re}(V)^{a}= & k A_{b}^{a} \operatorname{Im}(V)^{b}+k_{b} F_{c d}^{a b} \operatorname{Im}(V)^{c} \operatorname{Re}(V)^{d}+\operatorname{Re}(V)^{b} \mathfrak{L}_{\operatorname{Re}(V)} \mathcal{N}_{b}^{a} \\
& +\left[\operatorname{Re}(V)^{a}, \operatorname{Im}(V)^{a}\right]+\operatorname{Re}\left(\delta V^{a}(\mathrm{H}-\mathrm{L})\right) \\
\text { Blue }: \partial_{t} \operatorname{Im}(V)^{a}= & \kappa C_{b}^{a} \operatorname{Re}(V)^{b}+\kappa_{b} G_{c d}^{a b} \operatorname{Im}(V)^{c} \operatorname{Re}(V)^{d}+\operatorname{Im}(V)^{b} \mathfrak{L}_{\operatorname{Im}(V)} \mathcal{N}_{b}^{a} \\
& +\left[\operatorname{Im}(V)^{a}, \operatorname{Re}(V)^{a}\right]+\operatorname{Im}\left(\delta V^{a}(\mathrm{H}-\mathrm{L})\right)
\end{aligned}
$$

Secondly, from the split real-Red and imaginary-Blue vectorfields, $\partial_{t} \operatorname{Re}(V)^{a}$ and $\partial_{t} \operatorname{Im}(V)^{a}$ in (3), we can directly compose the following single ${ }^{1}$ It is clear that we can reverse this formal representation of the Red and Blue forces, so that the Blue manifold $M_{\text {Blue }}$ admits the tangent bundle $T M_{\text {Blue }}$ and the Red manifold admits the cotangent bundle $T^{*} M_{\text {Red }}$. 
complex-valued vectorfield, $\partial_{t} V^{a}\left(z^{a}, t\right): \mathcal{K} \rightarrow \mathbb{C}$, as a unique description of the battlefield dynamics:

$$
\partial_{t} V^{a}=k \mathcal{A}_{b}^{a} V^{b}+k_{b} \mathcal{F}_{c d}^{a b} V^{c} V^{d}+V^{b} \mathfrak{L}_{V} \mathcal{N}_{b}^{a}+\left[\operatorname{R}-\mathrm{I}(V)^{a}, \mathrm{I}-\mathrm{R}(V)^{a}\right]+\delta V^{a}(\mathrm{H}-\mathrm{L}),
$$

where $V^{a}\left(z^{a}, t\right)=R^{a}\left(x^{a}, t\right)+\mathrm{i} B^{a}\left(q^{a}, t\right) \in \mathcal{K}^{a}$ is the unique complex vector. Its time derivative, $\partial_{t} V^{a}\left(z^{a}, t\right)$, is our main actor, the battle-vectorfield, defined as the mapping from the Kähler battle-manifold $\mathcal{K}$ to the complex plane $\mathbb{C}$ :

$$
\partial_{t} V^{a}\left(z^{a}, t\right)=\partial_{t} R^{a}\left(x^{a}, t\right)+\mathrm{i} \partial_{t} B^{a}\left(q^{a}, t\right): \mathcal{K} \rightarrow \mathbb{C} .
$$

The battle-vectorfield $\partial_{t} V^{a}\left(z^{a}, t\right)$, defined by the complex-valued system of tensor differential Equations (4), represents a dynamical game played on the Kähler battle-manifold $\mathcal{K}$, in which the actors are the following complex tensors:

- $\mathcal{A}_{b}^{a}=A_{b}^{a}+\mathrm{i} C_{b}^{a} \in \mathcal{K}$,

- $\mathcal{F}_{c d}^{a b}=F_{c d}^{a b}+\mathrm{i} G_{c d}^{a b} \in \mathcal{K}$,

- $\mathfrak{L}_{V} \mathcal{N}_{b}^{a}=\mathfrak{L}_{\operatorname{Re}(V)} \mathcal{N}_{b}^{a}+\mathrm{i} \mathfrak{L}_{\operatorname{Im}(V)} \mathfrak{N}_{b}^{a} \in \mathcal{K}$,

- $\left[\operatorname{R-I}(V)^{a}, \mathrm{I}-\mathrm{R}(V)^{a}\right]=\left[\operatorname{Re}(V)^{a}, \operatorname{Im}(V)^{a}\right]+\mathrm{i}\left[\operatorname{Im}(V)^{a}, \operatorname{Re}(V)^{a}\right] \in \mathcal{K}$, and

- $\delta V^{a}(\mathrm{H}-\mathrm{L})=\operatorname{Re}\left[\delta V^{a}(\mathrm{H}-\mathrm{L})\right]+\mathrm{i} \operatorname{Im}\left[\delta V^{a}(\mathrm{H}-\mathrm{L})\right] \in \mathcal{K}$.

The promised rigorous geometric underpinning of the battle-vectorfield $\partial_{t} V^{a}\left(z^{a}, t\right) \in \mathcal{K}$, defined by (4), is provided by the Kähler-Ricci (KR) flow, a geometric-dynamics structure defined on the Kähler battle-manifold $\mathcal{K} \equiv(\mathcal{K}, g) \equiv(\mathcal{K}, \omega)$ in the following four equivalent ways:

1) Globally, in terms of the Kähler form $\omega=\omega(t)$ and the Ricci curvature form $\operatorname{Ric}[\omega(t)]$, as:

$$
\partial_{t} \omega(t)=-\operatorname{Ric}[\omega(t)], \quad \omega(0)=\omega_{0}
$$

2) Locally, in terms of the Kähler metric tensor $g_{\overline{i j}}=g_{\overline{i j}}(t)$ and the Ricci curvature tensor $R_{\overline{i j}}=R_{\overline{i j}}(t)$, as:

$$
\partial_{t} g_{\overline{i j}}(t)=g_{\overline{i j}}(t)-R_{\overline{i j}}(t), \quad g_{\overline{i j}}(0)=g_{0} .
$$

3) In terms of the Kähler potential $\varphi=\varphi(t)$ and volume forms $\left(\omega^{n}, \omega_{\varphi}^{n}\right)$ as:

$$
\partial_{t} \varphi(t)=\varphi(t)+\log \left(\omega_{\varphi}^{n} / \omega^{n}\right)-g(t), \quad \varphi(0)=\varphi_{0} .
$$

4) In the form of the Monge-Ampere equation (with Dolbeault's $(\partial, \bar{\partial})$ -operators and the Kähler class condition, $\left.\omega_{0}+\mathrm{i} \partial \bar{\partial} \varphi>0\right)$ :

$$
\partial_{t} \varphi(t)=\log \left[\left(\omega_{0}+\mathrm{i} \partial \bar{\partial} \varphi\right)^{n} / \omega^{n}\right], \quad \varphi(0)=\varphi_{0} .
$$

The solutions of these four $\mathrm{KR}$ equations are called the KR solitons. They uniquely exist in the case of Kähler-Einstein metric: $R_{i \bar{j}}=\lambda g$, for some real constant $\lambda$. KR solitons can be threefold: shrinking (if $\lambda>0$ ), steady (if $\lambda=0$ ), or expanding (if $\lambda<0$ ). For more technical details on the Kähler-Ricci 
flow, see Appendix 1.2.

In summary, the proposed Red-Blue combat dynamics model:

$$
\partial_{t} V^{a}\left(z^{a}, t\right)=\partial_{t} R^{a}\left(x^{a}, t\right)+\mathrm{i} \partial_{t} B^{a}\left(q^{a}, t\right)
$$

is defined on the Kähler battle-manifold $\mathcal{K}$ by a single battle-vectorfield:

$$
\partial_{t} V^{a}=k \mathcal{A}_{b}^{a} V^{b}+k_{b} \mathcal{F}_{c d}^{a b} V^{c} V^{d}+V^{b} \mathfrak{L}_{V} \mathcal{N}_{b}^{a}+\left[\operatorname{R}-\mathrm{I}(V)^{a}, \mathrm{I}-\mathrm{R}(V)^{a}\right]+\delta V^{a}(\mathrm{H}-\mathrm{L}),
$$

which is underpinned by the geometric Kähler-Ricci flow on $\mathcal{K}$ :

$$
\partial_{t} \omega(t)=-\operatorname{Ric}[\omega(t)] \text {. }
$$

Since the Kähler-Ricci flow $\partial_{t} \omega(t)$ has threefold solitary solutions: shrinking, steady and expanding (depending on the parameters), by analogy, we conjecture that the battle-vectorfield $\partial_{t} V^{a}\left(z^{a}, t\right)$ also has solitary solutions of shrinking, steady and expanding nature. Therefore, the battle dynamics can be shrinking, steady, or expanding - as expected from the classical warfare analysis.

\subsection{Physics of Warfare: Lagrangian and Hamiltonian Structures of the Red and Blue Forces}

Now we give a physical interpretation of warfare, using geometric insights from the Kähler dynamics provided above (and in the Appendix 1). The Kähler battle-manifold $(\mathcal{K}, g)$, with the fundamental complex structure defined by its Hermitian metric $g=g_{R}+\mathrm{i} \omega_{S}$, includes the Riemannian structure $g_{R}$ (for the Red force) and the symplectic structure $\omega_{S}$ (for the Blue force)—or vice versa. The Riemannian structure, $g_{R}=g_{a b} \mathrm{~d} x^{a} \mathrm{~d} x^{b} \in T M_{\text {Red }}$, naturally admits Lagrangian dynamics for the Red force, derived from the Lagrangian energy function, $L(x, \dot{x}): T M_{\text {Red }} \rightarrow \mathbb{R} ;$ the symplectic structure, $\omega_{S}=d p_{a} \wedge d q^{a} \in T^{*} M_{\text {Blue }}$, naturally admits Hamiltonian dynamics for the Blue force, derived from the Hamiltonian energy function, $H(q, p): T^{*} M_{\text {Blue }} \rightarrow \mathbb{R}-$ or vice versa.

Next, we recall that general forced-and-dissipative mechanics (see, e.g. [5] and the references therein) in Lagrangian form reads:

$$
\dot{L}_{\dot{x}^{a}}+\Phi_{\dot{x}^{a}}=L_{x^{a}}+F_{a},
$$

and in Hamiltonian form reads:

$$
\dot{q}^{a}=H_{p_{a}}-\Phi_{p_{a}}, \quad \dot{p}_{a}=F_{a}-H_{q^{a}}+\Phi_{q^{a}},
$$

where new $(x, p, q)$-indices denote partial derivatives (which is common with PDEs), $F_{a}$ represents the covector of generalized external forces and the scalar function $\Phi$, given by the mappings $\Phi(\dot{x}): T M_{\text {Red }} \rightarrow \mathbb{R}$ (for Lagrangian mechanics) and $\Phi(q, p): T^{*} M_{\text {Blue }} \rightarrow \mathbb{R}$ (for Hamiltonian mechanics)

represents Rayleigh's dissipation function (describing internal frictional forces proportional to velocity).

So, let us try to formally match the Red and Blue vectorfields from Equations (1) with the general Lagrangian Equations (5) and the general Hamiltonian Equations (6): 


$$
\begin{aligned}
& \dot{L}_{\dot{x}^{a}}+\Phi_{\dot{x}^{a}}=L_{x^{a}}+F_{a} \Leftrightarrow \\
& \operatorname{Red}: \dot{R}^{a}=c A_{b}^{a} B^{b}+c_{b} F_{c d}^{a b} B^{c} R^{d}+R^{b} \mathfrak{L}_{R} U_{b}^{a}+\left[R^{a}, B^{a}\right]+\delta R^{a}(\mathrm{H}-\mathrm{L}),
\end{aligned}
$$

and

$$
\begin{aligned}
& \dot{q}^{a}=H_{p_{a}}-\Phi_{p_{a}}, \quad \dot{p}_{a}=F_{a}-H_{q^{a}}+\Phi_{q^{a}} \Leftrightarrow \\
& \text { Blue }: \dot{B}^{a}=\kappa C_{b}^{a} R^{b}+\kappa_{b} G_{c d}^{a b} B^{c} R^{d}+B^{b} \mathfrak{L}_{B} W_{b}^{a}+\left[B^{a}, R^{a}\right]+\delta B^{a}(\mathrm{H}-\mathrm{L}) .
\end{aligned}
$$

By comparing the general forced-and-dissipative mechanics with our Red and Blue vectorfields, we make the following two observations. Firstly, we can see that there are no any covectors of external forces $F_{a}$ in the Red and Blue (pure velocity) vectorfields, so we can reduce our matches to:

$$
\begin{aligned}
& \dot{L}_{\dot{x}^{a}}+\Phi_{\dot{x}^{a}}=L_{x^{a}} \Leftrightarrow \\
& \operatorname{Red}: \dot{R}^{a}=c A_{b}^{a} B^{b}+c_{b} F_{c d}^{a b} B^{c} R^{d}+\left[R^{a}, B^{a}\right]+R^{b} \mathfrak{L}_{R} U_{b}^{a}+\delta R(\mathrm{H}-\mathrm{L})^{a},
\end{aligned}
$$

and

$$
\begin{aligned}
& \dot{q}^{a}=H_{p_{a}}-\Phi_{p_{a}}, \quad \dot{p}_{a}=-H_{q^{a}}+\Phi_{q^{a}} \quad \Leftrightarrow \\
& \text { Blue }: \dot{B}^{a}=\kappa C_{b}^{a} R^{b}+\kappa_{b} G_{c d}^{a b} B^{c} R^{d}+\left[B^{a}, R^{a}\right]+B^{b} \mathfrak{L}_{B} W_{b}^{a}+\delta B(\mathrm{H}-\mathrm{L})^{a} .
\end{aligned}
$$

Secondly, since the Red and Blue vectorfields are generalized from classical Lanchester equations (which are the 1st-order ODEs), there are no any covectors of inertial (internal) forces either. In other words, the Red and Blue vectorfields physically correspond to dynamics of highly viscous/dissipative fluids, in which inertial forces can be neglected, so we can make the second reduction as:

$$
\begin{aligned}
& \Phi_{\dot{x}^{a}}=L_{x^{a}} \Leftrightarrow \\
& \text { Red : } \dot{R}^{a}=c A_{b}^{a} B^{b}+c_{b} F_{c d}^{a b} B^{c} R^{d}+\left[R^{a}, B^{a}\right]+R^{b} \mathfrak{L}_{R} U_{b}^{a}+\delta R(\mathrm{H}-\mathrm{L})^{a},
\end{aligned}
$$

and

$$
\begin{aligned}
& \dot{q}^{a}=H_{p_{a}}-\Phi_{p_{a}} \Leftrightarrow \\
& \text { Blue : } \dot{B}^{a}=\kappa C_{b}^{a} R^{b}+\kappa_{b} G_{c d}^{a b} B^{c} R^{d}+\left[B^{a}, R^{a}\right]+B^{b} \mathfrak{L}_{B} W_{b}^{a}+\delta B(\mathrm{H}-\mathrm{L})^{a} .
\end{aligned}
$$

Therefore, since our Red and Blue vectorfields are pure velocity-vectorfields without internal or external force co-vectorfields, both Lagrangian and Hamiltonian equations are reduced to the 1st-order systems of ODEs: in Lagrangian formulation the 2nd-order (inertial force) term vanishes, and in Hamiltonian formulation the whole force equation vanishes (momenta still exist but their time derivatives vanish).

\section{Computational Wargame: "Entropy Battle"}

The computational wargame called the Entropy Battle (see Figure 1) is currently being implemented in C\# language (on. Net 4.7), using Irrlicht 3D graphics engine and Bullet $3 \mathrm{D}$ physics engine, and implementing the metaphysics of wargaming outlined in the next subsection. The core version of the wargame simulates the aircraft battle scenario from [4] and [1] with 30 aircraft on each 


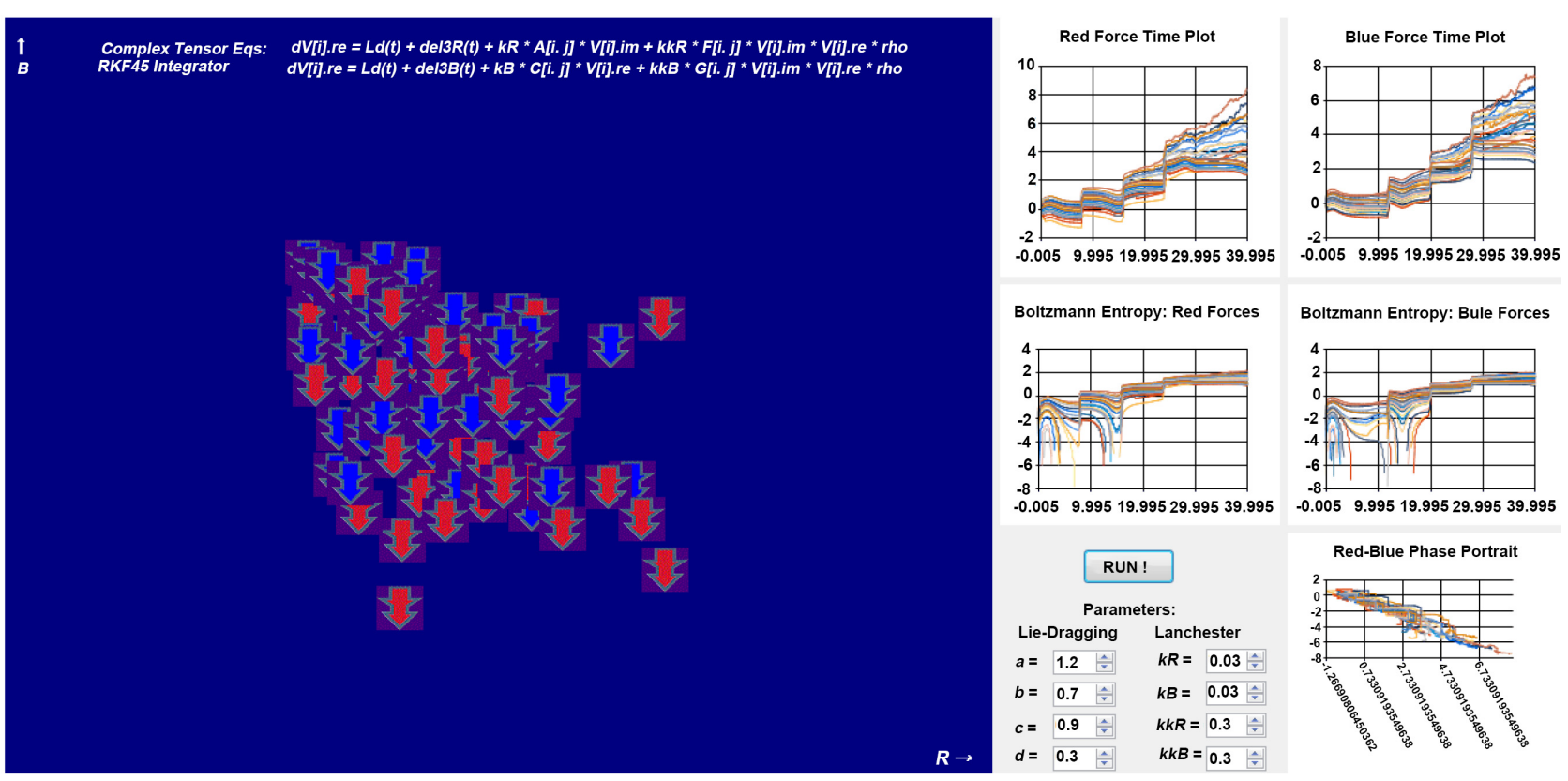

Figure 1. A prototype of the computational TCW-wargame called the Entropy Battle. The wargame is currently under development in the Joint and Operations Analysis Division, Defence Science \& Technology Group, Australia, using the C\# language, with Irrlicht 3D graphics engine and Bullet 3D physics engine. It implements a simplified version of the battle-vectorfield $\partial_{t} V^{a}\left(z^{a}, t\right)$, moving predominantly in the horizontal complex plane, solved by the complex-valued RKF45 (Cash-Karp) integrator and implementing the general metaphysics of wargaming.

side: Red aircraft starts in the bipartite formation and Blue aircraft starts in the tripartite formation. The battle is formally defined as a simplified version of the battle-vectorfield $\partial_{t} V^{a}\left(z^{a}, t\right)$, moving/flying predominantly in the horizontal complex plane $\mathbb{C}$. It is numerically solved in adaptive time steps using the complex-valued RKF45 (Cash-Karp) integrator, which is fast, accurate and almost symplectic.

The extended version of the Entropy Battle wargame has two levels:

Top level is the core aircraft battle, and

Bottom level has two scenarios (both formally defined by a simplified version of the battle-vectorfield $\partial_{t} V^{a}\left(z^{a}, t\right)$ moving in the complex plane):

- Land battle between Red and Blue land vehicles, and

- Sea battle between Red and Blue boats.

In both cases, the Entropy Battle wargame follows the general metaphysics of wargaming outlined as follows.

\section{Metaphysics of Wargaming: Warfare Entropy and "Combat Sig- natures" in the Battlespace}

- The stage for combat dynamics is the Red-Blue battlespace, which can be modeled by a dynamical concept of the phase-space. From a bird-view (or, from God's Eye), the phase-space reduces to its 2D order-parameter subspace, the Red-Blue phase-plane, which is usually used in simulations.

- The concept of the phase-space (in our case spanned by the Red and Blue 
forces) comes from Hamiltonian mechanics (when W.R. Hamilton formally unified Lagrangian mechanics and optics). It is also used in statistical mechanics. Besides, the 2D phase plane was the main analytical tool of $\mathrm{H}$. Poincaré in his qualitative analysis of differential equations, from which both topology and chaos theory emerged. Finally, L. Boltzmann defined the entropy by coarse-graining the phase space. Every kind of entropy (including Boltzmann, Gibbs, Shannon, Kolmogorov-Sinai, Rényi, Bekenstein-Hawking, Kosko fuzzy, entanglement, topological, partition-function based, path-integral based, etc.) is essentially a logarithm of some more fundamental underlying (probabilistic, phase space or topological) measure, therefore it is itself an additive measure, which in our combat case gives:

Total combat entropy $=$ Red-entropy + Blue-entropy.

- The cornerstone of Hamiltonian and statistical mechanics (as well as ergodic dynamics) is the key concept related to the Warfare Entropy and "Combat Signatures" in the Battlespace. It is the famous Liouville's theorem: The flow of a conservative Hamiltonian vectorfield preserves the phase-space Volume (technically, Hamiltonian flow is a symplectomorphism: the Lie derivative of the volume form: $d R e d^{\wedge} d B l u e$ along the [Red, Blue] vectorfield vanishes). This volume preservation necessarily implies various shape distortions (called "combat signatures") and therefore uncertainty!

- Liouville's theorem-based interpretation of the Warfare Entropy and "Combat Signatures": If dynamics in the Red-Blue phase-plane stretches in the Red direction, it necessarily shrinks in the Blue direction, and vice versa. The stretching and shrinking distortions of the Combat Area cause rapid entropy growth and combat signatures in the $2 \mathrm{D}$ phase-plane. More generally, in higher Red-Blue phase-space dimensions, Liouville's theorem causes Hamiltonian chaos, because there are so many possible ways for stretching and shrinking, each one reflected by entropy growth. There is no chaos in the $2 \mathrm{D}$ phase plane (theorem), but the entropy growth is still observable, since, e.g., the Kolmogorov-Sinai entropy is a sum of all Lyapunov exponents (both positive-chaotic and negativenonchaotic).

- We can assume that, at least in a short time interval, the Red-Blue combat dynamics in the battlespace is conservative (no energy sources or sinks). Therefore, for a short time period, all combat dynamics can be derived from the so-called battle Hamiltonian (total combat energy function in an isolated region of battlespace) - at a certain entropy level. In the next short time period, we again have the conservative combat dynamics-at a higher entropy level, etc.

- Generalization/relaxation of Liouville's theorem: the so-called HamiltonLangevin framework has been proposed in [3], to include: delta-strikes, dissipation and random external forces. If the magnitude of these non- 
conservative influences is not overwhelming, the entropic stretchingand-shrinking effect of Liouville's theorem is still visible.

- While slow changes of the battlefield are governed by Liouville's theorem, fast changes are governed by Onsager ${ }^{2}$ - Prigogine's ${ }^{3}$ entropic, irreversible, non-equilibrium thermodynamics with the arrow-of-time. ${ }^{4}$ Sudden entropy growths in open combat Red-Blue systems reflect sudden energy dissipations due to impulsive Red-Blue crashes.

- In summary, general combat dynamics and wargaming necessarily includes both the reversible Hamiltonian-type dynamics (governed by Liouville's theorem) and irreversible Prigogine's non-equilibrium thermodynamics of open systems (exhibiting rapid entropy growth).

\section{Conclusion}

We have presented the Kähler dynamics approach to battlefields. It is the complex-dynamics synthesis of the combat dynamics series called the tensor-centric warfare, which includes tensor generalization of classical Lanchester-type combat equations, entropic Lie-dragging for modeling warfare uncertainty and symmetry, various (both deterministic and random) delta-strikes and missiles, and deep-learning at the battlefield. This synthesis is performed in the form of the complex battle-vectorfield, defined using the global framework of Kähler battle-manifolds. The proposed Red-Blue combat dynamics model is defined on the Kähler battle-manifold by a unique battle-vectorfield which is underpinned by the geometric Kähler-Ricci flow. This complex synthesis gives a new insight into the "physics of warfare" in terms of "hidden" Lagrangian and Hamiltonian structures of the battlefields. It also provides a convenient and efficient computational framework for entropic wargaming, in which the Entropy Battle is currently under development.

\section{Acknowledgements}

The authors are grateful to Dr. Tim McKay and Dr. Brandon Pincombe, Joint and Operations Analysis Division, Defence Science \& Technology Group, Australia-for their constructive comments which have improved the quality of this paper.

\section{Conflicts of Interest}

The authors declare no conflicts of interest regarding the publication of this paper.

\footnotetext{
${ }^{2}$ Lars Onsager, 1968 Nobel Prize in Chemistry. ${ }^{3}$ Ilya Prigogine, 1977 Nobel Prize in Chemistry.

${ }^{4}$ When a glass or an egg is dropped on the floor, the arrow-of-time is obvious-once a glass or an egg is broken, there is no way to collect it back to its original state. However, all equations of classical and quantum physics are time-reversible, so they cannot model this simple crash-dissipation event. The only adequate physical/chemical principle at work is Onsager-Prigogine's Extended Second Law of Thermodynamics, reflecting the sudden entropy growth (burst) due to a dissipative crash.
} 


\section{References}

[1] Ivancevic, V., Pourbeik, P. and Reid, D. (2018) Tensor-Centric Warfare I: Tensor Lanchester Equations. Intelligent Control and Automation, 9, 11-29. https://doi.org/10.4236/ica.2018.92002

[2] Ivancevic, V., Reid, D. and Pourbeik, P. (2018) Tensor-Centric Warfare II: Entropic Uncertainty Modeling, Intelligent Control and Automation, 9, 30-51.

[3] Ivancevic, V., Pourbeik, P. and Reid, D. (2018) Tensor-Centric Warfare III: Combat Dynamics with Delta-Strikes. Intelligent Control and Automation, 9, 107-122.

[4] McLemore, C., Gaver, D. and Jacobs, P. (2016) Model for Geographically Distributed Combat Interactions of Swarming Naval and Air Forces. Naval Research Logistics, 63, 562-576. https://doi.org/10.1002/nav.21720

[5] Ivancevic, V. (2017) Kähler Dynamics for the Universal Multi-Robot Fleet. Journal of Geometry and Symmetry in Physics, 45, 67-85. https://doi.org/10.7546/jgsp-45-2017-67-85

[6] Ivancevic, V., Reid, D. and Scholz, J. (2014) Action-Amplitude Approach to Controlled Entropic Self-Organization. Entropy, 16, 2699-2712.

https://doi.org/10.3390/e16052699

[7] Ivancevic, V. and Reid, D. (2015) Complexity and Control: Towards a Rigorous Behavioral Theory of Complex Dynamical Systems. World Scientific, Singapore.

[8] Ivancevic, V., Reid, D. and Pilling, M. (2017) Mathematics of Autonomy: Mathematical Methods for Cyber-Physical-Cognitive Systems. World Scientific, New Jersey/Singapore. https://doi.org/10.1142/10716

[9] Griffiths, P. and Harris, J. (1978) Principles of Algebraic Geometry, Pure and Applied Mathematics. Wiley-Interscience, New York.

[10] Nakahara, M. (2003) Geometry, Topology and Physics. 2nd Edition, IOP, Bristol and Philadelphia.

[11] Donaldson, S.K. (1999) Symmetric Spaces, Kähler Geometry and Hamiltonian Dynamics. American Mathematical Society Translations: Series 2, 196, 13-33. https://doi.org/10.1090/trans2/196/02

[12] Song, J. and Weinkove, B. (2012) Lecture Notes on the Kähler-Ricci Flow. arXiv:1212.3653, 94.

[13] Yau, S.T. (2006) Structure of Three-Manifolds-Poincaré and Geometrization Conjectures. Morningside Center of Mathematics, Beijing.

[14] Cao, H.-D. (2012) The Kähler-Ricci Flow on Fano Manifolds. arXiv:1212.6227.

[15] He, W. (2015) On the Space of Kähler Potentials. Communications on Pure and Applied Mathematics, 68, 332-343. https://doi.org/10.1090/trans2/196/02

[16] Calabi, E. and Vesentini, E. (1960) On Compact, Locally Symmetric Kähler Manifolds. Annals of Mathematics, 71, 472-507. https://doi.org/10.2307/1969939

[17] Yau, S.T. (1978) On the Ricci Curvature of a Compact Kähler Manifold and the Complex Monge-Ampere Equation. Communications on Pure and Applied Mathematics, 31, 339-441. https://doi.org/10.1002/cpa.3160310304

[18] Ivancevic, V. and Ivancevic, T. (2011) Ricci Flow and Nonlinear Reaction-Diffusion Systems in Biology, Chemistry, and Physics. Nonlinear Dynamics, 65, 35-54. https://doi.org/10.1007/s11071-010-9872-6

[19] Hamilton, R.S. (1982) Three-Manifolds with Positive Ricci Curvature. Journal of Differential Geometry, 17, 255-306. https://doi.org/10.4310/jdg/1214436922 
[20] Hamilton, R.S. (1986) Four-Manifolds with Positive Curvature Operator. Journal of Differential Geometry, 24, 153-179. https://doi.org/10.4310/jdg/1214440433

[21] Hamilton, R.S. (1988) The Ricci Flow on Surfaces. Contemporary Mathematics, 71, 237-261,

[22] Tian, G. and Zhang, Z. (2013) Regularity of Kähler-Ricci Flows on Fano Manifolds. arXiv:1310.5897 [math.DG].

[23] Song, J., Phong, D.H. and Sturm, J. (2012) Complex Monge-Ampere Equations. Surveys in Differential Geometry, 17, 327-411. 


\section{Appendix: Kähler Manifolds and Kähler-Ricci Flow}

In this section, we give a brief review of Kähler manifolds (the main reference is [9]) and the Kähler-Ricci flow on them, which constitutes the geometric framework for the complex Red-Blue battle-vectorfield.

\subsection{Geometry and Dynamics of Kähler Manifolds}

Let $\mathcal{K}=\mathcal{K}^{n}$ be a compact (i.e., closed and bounded) complex $n$-manifold ${ }^{5}$ of complex dimension $n$ (see [9] [10]). To be able to write various tensors on the manifold $\mathcal{K}$, we chose a local point $p \in \mathcal{K}$ with the neighborhood chart $U$ that includes: 1) the holomorphic coordinates and their complex-conjugates: $\left.\left\{z^{i}=x^{i}+\mathrm{i} y^{i}, \bar{z}^{i}=\bar{x}^{i}+\overline{\mathrm{y}}^{i}\right\} \in U_{p} \subset \mathcal{K}, 2\right)$ the natural basis of vectorfields in the tangent space $T_{p} \mathcal{K}$ at $p:\left\{\partial_{i}, \partial_{\bar{i}}\right\} \in T_{p} \mathcal{K}$ (using $\partial_{i} \equiv \partial / \partial z^{i}$ ), and 3 ) the dual basis of co-vectorfields (i.e., holomorphic 1 -forms) ${ }^{6}$ in the cotangent space $T_{p}^{*} \mathcal{K}$ at $p:\left\{d z^{i}, d \bar{z}^{i}\right\} \in T_{p}^{*} \mathcal{K}$.

To make the complex manifold $\mathcal{K}=(\mathcal{K}, g)=(\mathcal{K}, \omega)$ into a Kähler $n$-manifold, we need to specify on it a Kähler metric $g$ and its associated Kähler form $\omega$, as follows (compare with [11] [12] [13] [14]). Consider a Hermitian metric $g$ defined at each point $\left\{z^{i}, \bar{z}^{i}\right\} \in \mathcal{K}$ by a smooth positive-definite $(1,1)$-tensor field $g_{\overline{i j}}=g_{\overline{i j}}\left(z^{i}, \bar{z}^{j}\right)$, for $i, j=1, \cdots, n$, as: ${ }^{8}$

${ }^{5}$ Recall that a complex-valued function $f: \mathbb{C}^{n} \rightarrow \mathbb{C}$ is called holomorphic if-and-only-if (iff) $f=f_{1}+\mathrm{i} f_{2}$ satisfies the Cauchy-Riemann relations for each holomorphic coordinate, $z^{j}=x^{j}+\mathrm{i} y^{j}:$

$$
\partial_{x^{\prime}} f_{1}=\partial_{y^{\prime}} f_{2}, \quad \partial_{x^{\prime}} f_{2}=-\partial_{y^{\prime}} f_{1}, \quad\left(\partial_{x^{\prime}} \equiv \partial / \partial x^{j}\right) .
$$

A complex manifold $\mathcal{K}$ (like $\mathbb{C}^{n}$ and Riemann surfaces) is a manifold with an atlas consisting of charts $t_{i j}: U_{i} \cap U_{j} \rightarrow \mathbb{C}^{n}$, such that the transition functions $t_{i j}(z)$ are holomorphic and satisfy the cocycle condition: $t_{i k}(z)=t_{i j}(z) t_{j k}(z)$ on triple overlaps $U_{i} \cap U_{j} \cap U_{k}$. An almost complex structure $J$ is defined on a complex manifold $\mathcal{K}$ as:

$$
J \partial_{z^{\prime}}=\mathrm{i} \partial_{z^{\prime}}, \quad J \partial_{\bar{z}^{\prime}}=-\mathrm{i} \partial_{\bar{z}^{\prime}}, \quad J^{2}=-1 .
$$

${ }^{6}$ The basis 1-forms (co-vectorfields) $\left\{d z^{i}, d \bar{z}^{i}\right\}$ are holomorphic de Rham differentials of the holomorphic coordinates:

$$
d z^{i}=d x^{i}+\mathrm{i} d y^{i} \quad \text { and } \quad d \bar{z}^{i}=d x^{i}-\mathrm{i} d y^{i} .
$$

They induce the holomorphic velocities:

$$
\dot{z}^{i}=\dot{x}^{i}+i \dot{y}^{i} \quad \text { and } \quad \dot{\bar{z}}^{i}=\dot{x}^{i}-\dot{\mathrm{i}}^{i} \text {. }
$$

${ }^{7} \mathrm{~A}$ Hermitian manifold $(\mathcal{K}, g)$ is a complex manifold $\mathcal{K}$ with a Hermitian metric tensor $g_{\overline{i j}}=g_{\overline{i j}}\left(z^{i}, \bar{z}^{j}\right)$, such that $\left(g^{\bar{j}}\right)$ is a positive-definite Hermitian matrix function,

$\left(g^{\bar{j}}\right)=\left(g^{\bar{j}}\right)^{*}>0$. A Hermitian manifold $(\mathcal{K}, g)$ becomes a Kähler manifold iff the almost complex structure $J$ on it satisfies the condition: $\nabla_{k} J=0$, where $\nabla_{k}$ is the Levi-Civita connection on $(\mathcal{K}, g)$, defined later in this section.

${ }^{8}$ The metric $g$ given by (7) has the following dynamical interpretation: it defines the complex kinetic-energy Lagrangian $L(\dot{z}, \dot{\bar{z}}): \mathcal{K} \rightarrow \mathbb{C}$, in holomorphic coordinates $\left\{z^{i}, \bar{z}^{i}\right\} \in \mathcal{K}$ given as:

$$
L(\dot{z}, \dot{\bar{z}})=\frac{1}{2} g_{i \bar{j}} \dot{z}^{i} \otimes \dot{\bar{z}}^{j},
$$

from which the conservative Lagrangian equations read in the contravariant form:

$$
\ddot{z}^{i}(t)+\Gamma_{j k}^{\bar{i}} \dot{z}^{j}(t) \dot{\bar{z}}^{k}(t)=0,
$$

and in the standard covariant form (with indices denoting partial derivatives):

$$
\dot{L}_{z^{t}}-L_{z^{i}}=0 \text {. }
$$




$$
g=g_{\overline{i j}} d z^{i} \otimes d \bar{z}^{j}>0
$$

such that $\left(g_{\overline{i j}}\right)$ is a positive-definite Hermitian matrix. Its inverse $g^{\bar{j}}$ is given by the matrix $\left(g^{\bar{j}}\right)=\left(g_{\overline{i j}}\right)^{-1}$. Associated to the Hermitian metric $g$, there is a real positive-definite exterior $(1,1)$-form $\omega=\omega_{\overline{i j}}\left(z^{i}, \bar{z}^{j}\right)$ on $\mathcal{K}$, defined by: ${ }^{9}$

$$
\omega=\mathrm{i} g_{\overline{i j}} d z^{i} \wedge d \bar{z}^{j}>0 .
$$

If the form $\omega$ is closed, $d \omega=0$, then $g$ is called the Kähler metric and $\omega$ is called the Kähler form. The fundamental closure condition: $d \omega=0$ is called the Kähler condition, the global condition for any Kähler manifold $\mathcal{K}$, which is locally in $\left(z^{i}, \bar{z}^{j}\right) \in U \subset \mathcal{K}$ equivalent to the following metric symmetries: ${ }^{10}$

$$
\partial_{j} g_{i \bar{k}}=\partial_{i} g_{j \bar{k}} \quad \text { and } \quad \partial_{\bar{j}} g_{k \bar{i}}=\partial_{\bar{i}} g_{\bar{k} \bar{j}}
$$

(independent of the choice of local holomorphic coordinates $\left.\left(z^{i}, \bar{z}^{j}\right) \in U \subset \mathcal{K}\right)$. In (9), $\partial_{j} \equiv \partial$ and $\partial_{\bar{j}} \equiv \bar{\partial}$ are Dolbeault's differential operators, which are the additive components of the exterior derivative (de Rham differential) $d$ on $\mathcal{K}: d=\partial+\bar{\partial} \cdot{ }^{11}$ In that case, as shown by E. Kähler ${ }^{9}$ From the Kähler form (8) with $\bar{z}^{i}$ as canonical momenta, the Hamiltonian formalism can be derived from the complex kinetic-energy Hamiltonian $H(z, \bar{z}): \mathcal{K} \rightarrow \mathbb{C}$ in holomorphic coordinates $\left\{z^{i}, \bar{z}^{i}\right\} \in \mathcal{K}$ given as:

$$
H(z, \bar{z})=\frac{1}{2} g_{i \bar{j}} d z^{i} \wedge d \bar{z}^{j}
$$

from which the conservative Hamiltonian equations read (with indices denoting partial derivatives):

$$
\dot{z}^{i}=H_{\bar{z}^{i}}, \quad \dot{\bar{z}}^{i}=-H_{z^{i}}
$$

${ }^{10}$ Since $d \omega=0$, we have:

$$
\begin{aligned}
0 & =(\partial+\bar{\partial}) \mathrm{ig}_{\overline{i j}} d z^{i} \wedge d \bar{z}^{j}=\mathrm{i} \partial_{k} g_{\overline{i j}} d z^{k} \wedge d z^{i} \wedge d \bar{z}^{j}+\mathrm{i} \partial_{\bar{k}} g_{\overline{i j}} d \bar{z}^{k} \wedge d z^{i} \wedge d \bar{z}^{j} \\
& =\frac{1}{2} \mathrm{i}\left(\partial_{k} g_{\overline{i j}}-\partial_{i} g_{\overline{k j}}\right) d z^{k} \wedge d z^{i} \wedge d \bar{z}^{j}+\frac{1}{2} \mathrm{i}\left(\partial_{\bar{k}} g_{\overline{i j}}-\partial_{\bar{j}} g_{i \bar{k}}\right) d \bar{z}^{k} \wedge d z^{i} \wedge d \bar{z}^{j},
\end{aligned}
$$

which implies:

$$
\partial_{j} g_{i \bar{k}}=\partial_{i} g_{j \bar{k}} \quad \text { and } \quad \partial_{\bar{j}} g_{k \bar{i}}=\partial_{\bar{i}} g_{\bar{k}} \text {. }
$$

${ }^{11}$ Recall that the exterior derivative, or de Rham differential, $d: \Omega^{p}(\mathcal{K}) \rightarrow \Omega^{p+1}(\mathcal{K})$ is the map on the space $\Omega^{p}(\mathcal{K})$ of exterior $p$-forms on the complex manifold $\mathcal{K}$, which generalizes standard gradient, curl and divergence operators from vector calculus. Being the additive components of the exterior derivative: $d=\partial+\bar{\partial}$, so that $d f=\partial f+\bar{\partial} f$ for any smooth real function $f$ on $\mathcal{K}$, Dolbeault's differential operators $(\partial, \bar{\partial})$ are the maps on the space $\Omega^{p, q}(\mathcal{K})$ of exterior forms on $\mathcal{K}$, such that $\partial: \Omega^{p, q}(\mathcal{K}) \rightarrow \Omega^{p+1, q}(\mathcal{K})$ and $\bar{\partial}: \Omega^{p, q}\left((\mathcal{K}) \rightarrow \Omega^{p, q+1}(\mathcal{K})\right.$. They play the major role in the famous " $\partial \bar{\partial}$-lemma" and the associated Dolbeault cohomology (from the Hodge theory). Any function $f: \mathcal{K} \rightarrow \mathbb{C}$ is holomorphic iff $\bar{\partial} f=0$ (the Cauchy-Riemann equations). In a local $z^{k}$ -coordinate chart $U \subset \mathcal{K}$, for any holomorphic function $f \in U,(\partial, \bar{\partial})$-operators are given by:

$$
\begin{array}{ll}
\partial f=\left(\partial_{x^{k}} f-\mathrm{i} \partial_{y^{k}} f\right) d z^{k}, & \bar{\partial} f=\left(\partial_{x^{k}} f-\mathrm{i} \partial_{y^{k}} f\right) d \bar{z}^{k}, \\
\text { such that }: \partial^{2}=0, \quad \bar{\partial}^{2}=0, & \partial \bar{\partial}+\bar{\partial} \partial=0 .
\end{array}
$$


himself in 1933, the metric tensor $g_{j \bar{k}}$ can be written in terms of a real-valued smooth function $\varphi: \mathcal{K} \rightarrow \mathbb{R}$, called the Kähler potential (see below), as: ${ }^{12}$

$$
g_{j \bar{k}}=\frac{\partial^{2} \varphi}{\partial z^{j} \partial z^{\bar{k}}} \equiv \partial_{j} \partial_{\bar{k}} \varphi \equiv \partial \bar{\partial} \varphi \Rightarrow \omega_{j \bar{k}}=\mathrm{i} \frac{\partial^{2} \varphi}{\partial z^{j} \partial z^{\bar{k}}} \equiv \mathrm{i} \partial_{j} \partial_{\bar{k}} \varphi \equiv \mathrm{i} \partial \bar{\partial} \varphi .
$$

We remark that the two differential expressions with the Kähler potential $\varphi,{ }^{13} g=\partial \bar{\partial} \varphi$ and $\omega=\mathrm{i} \partial \bar{\partial} \varphi$, both governed by the $\partial \bar{\partial}$-lemma (see below) constitute the core of the Kähler geometry, so that any other geometro-dynamical structure on $(\mathcal{K}, g)$, including the Kähler-Ricci flow and the Monge-Amperè equation, is derivable from them.

Holomorphic vectorfields and co-vectorfields (1-forms) are defined on $(\mathcal{K}, g)$ by their appropriate holomorphic coordinate transformations (or, diffeomorphisms) in $T_{p} \mathcal{K}$ and $T_{p}^{*} \mathcal{K}$, respectively. Let $v=v^{i} \partial_{i}$ and $u=u^{\bar{i}} \partial_{\bar{i}}$ be $T^{1,0}$ and $T^{0,1}$ vectorfields in $T_{p} \mathcal{K}$, such that $\partial_{\bar{j}} v^{i}=\partial_{j} u^{\bar{i}}=0$, and let $\alpha=\alpha_{i} d z^{i}$ and $\beta=\beta_{\bar{i}} d \overline{z^{i}}$ be $(1,0)$ and $(0,1)$ co-vectorfields in $T_{p}^{*} \mathcal{K}$, such that $\alpha_{i} d \bar{z}^{j}=\beta_{i} d z^{j}=0$. If $\left\{\tilde{z}^{i}\right\}=\left\{\tilde{z}^{1}, \cdots, \tilde{z}^{n}\right\}$ is another holomorphic coordinate system on $\mathcal{K}$, then on the overlap $\left\{z^{i}\right\} \cap\left\{\tilde{z}^{i}\right\} \in \mathcal{K}$ the following diffeomorphisms hold:

$$
\begin{aligned}
& v^{j}=v^{i} \frac{\partial \tilde{z}^{j}}{\partial z^{i}}, \quad u^{\bar{j}}=u^{\bar{i}} \frac{\overline{\partial \tilde{z}^{j}}}{\partial z^{i}} \\
& \tilde{a}_{j}=\alpha_{i} \frac{\partial z^{i}}{\partial \tilde{z}^{j}}, \quad \beta_{\bar{j}}=\beta_{\bar{i}} \frac{\overline{\partial z^{i}}}{\partial \tilde{z}^{j}} .
\end{aligned}
$$

The Kähler metric $g$ induces the Levi-Civita connection on $(\mathcal{K}, g)$, given by the Christoffel symbols $\Gamma_{j k}^{i}$ on $(\mathcal{K}, g)$, defined simply by: ${ }^{14}$

$$
\Gamma_{j k}^{i}=g^{\bar{m} i} \partial_{j} g_{k \bar{m}} .
$$

$\Gamma_{j k}^{i}$ are not the components of a tensor; however, if $g_{\overline{i j}}$ and $\hat{g}_{\overline{i j}}$ are two Kähler metrics with Christoffel's symbols $\Gamma_{j k}^{i}$ and $\hat{\Gamma}_{j k}^{i}$ then the difference $\Gamma_{j k}^{i}-\hat{\Gamma}_{j k}^{i}$ is a tensor. From the Kähler condition (9) it follows that $\Gamma_{j k}^{i}$ are symmetric in the lower indices: $\Gamma_{j k}^{i}=\Gamma_{k j}^{i}$.

Using Christoffel's symbols $\Gamma_{j k}^{i}$, we can defined the pair of covariant derivatives $\left(\nabla_{k}, \nabla_{\bar{k}}\right)$ on $(\mathcal{K}, g)$, which act on smooth functions $f$ on $\mathcal{K}$ as: $\nabla_{i} f=\partial_{i} f, \nabla_{\bar{i}} f=\partial_{\bar{i}} f$. On the vectorfields $(v, u)$ on $T \mathcal{K}$ and co-vectorfields

${ }^{12}$ Potential definition of the Kähler metric, $g_{\overline{i j}}=\partial \bar{\partial} \varphi$, implies that the Ricci curvature $\operatorname{Ric}(g)=R_{\overline{i j}}$ (see below) is given by:

$$
R_{\overline{i j}}=-\frac{\partial^{2} \varphi}{\partial z^{j} \partial z^{\bar{k}}} \log \left[\operatorname{det}\left(g_{\overline{i j}}\right)\right] \equiv-\partial_{j} \partial_{\bar{k}} \log \left[\operatorname{det}\left(g_{\overline{i j}}\right)\right] \equiv-\partial \bar{\partial} \log [\operatorname{det}(g)],
$$

and consequently, the solutions of the Einstein equation: Ric $=\lambda g$ (in components, $R_{\overline{i j}}=\lambda g_{\overline{i j}}$, for some real constant $\lambda$ ) can be defined by solving the scalar equation:

$$
\operatorname{det}(\partial \bar{\partial} \varphi)=\mathrm{e}^{-\lambda \varphi} .
$$

${ }^{13}$ For a real function $\varphi: \mathcal{K} \rightarrow \mathbb{R}$ the real $(1,1)$-form $\mathrm{i} \partial \bar{\partial} \varphi \equiv \mathrm{i} \partial_{j} \partial_{\bar{k}} \varphi$ is the complex Hessian of $\varphi$. ${ }^{14}$ The Christoffel symbols $\Gamma_{j k}^{i}$ on a Kähler manifold $\mathcal{K}$ do not have their conjugate part. Because of this unique feature of Kähler manifolds, the Riemann and Ricci curvature tensors (defined below) are much simpler on $\mathcal{K}$ than in standard Riemannian geometry. 
$(\alpha, \beta)$ on $T^{*} \mathcal{K}$, the covariant derivatives $\left(\nabla_{k}, \nabla_{\bar{k}}\right)$ act in the following way: ${ }^{15}$

$$
\begin{aligned}
& \nabla_{k} v^{i}=\partial_{k} v^{i}+\Gamma_{j k}^{i} v^{j}, \quad \nabla_{\bar{k}} v^{i}=\partial_{\bar{k}} v^{i}, \\
& \nabla_{k} u^{\bar{i}}=\partial_{k} u^{\bar{i}}, \quad \nabla_{\bar{k}} u^{\bar{i}}=\partial_{\bar{k}} u^{\bar{i}}+\overline{\Gamma_{j k}^{i}} u^{\bar{j}}, \\
& \nabla_{k} \alpha_{i}=\partial_{k} \alpha_{i}-\Gamma_{i k}^{j} \alpha_{j}, \quad \nabla_{\bar{k}} \alpha_{i}=\partial_{\bar{k}} \alpha_{i}, \\
& \nabla_{k} \beta_{\bar{i}}=\partial_{k} \beta_{\bar{i}}, \quad \nabla_{\bar{k}} \beta_{\bar{i}}=\partial_{\bar{k}} \beta_{\bar{i}}-\overline{\Gamma_{i k}^{j}} \beta_{\bar{j}} .
\end{aligned}
$$

In general, the Christoffel symbols $\Gamma_{j k}^{i}$ are chosen so that both covariant derivatives of the metric tensor vanish: $\nabla_{k} g_{\overline{i j}}=\nabla_{\bar{k}} g_{\overline{i j}}=0$. Similarly, a Hermitian manifold $(\mathcal{K}, g)$ is a Kähler manifold iff the almost complex structure $J$ satisfies: $\nabla_{k} J=\nabla_{\bar{k}} J=0$.

The Laplacian (or, rather Laplace-Beltrami) operator $\Delta$ is defined in local coordinates $\left(z^{i}, \bar{z}^{j}\right) \in \mathcal{K}$ as:

$$
\Delta \equiv \operatorname{det}\left(g_{\overline{i j}}\right)^{-\frac{1}{2}} \partial_{i}\left(\operatorname{det}\left(g_{\overline{i j}}\right)^{\frac{1}{2}} g^{\bar{j}} \partial_{\bar{j}}\right) .
$$

$\Delta$-action on smooth functions $f \in \mathcal{K}$ is given by:

$$
\Delta f=g^{\overline{j i}} \partial_{i} \partial_{\bar{j}} f=\operatorname{Tr}(\mathrm{i} \partial \bar{\partial} f),
$$

where $\operatorname{Tr}(\cdot)=\operatorname{Tr}_{\omega}(\cdot)$ is the trace operator (i.e., contraction with $\left.g^{\overline{j i}}\right) .{ }^{16}$ More generally, $\Delta$-action on an arbitrary tensor $T$ is defined in normal coordinates ${ }^{17}$ for $g$ on $(\mathcal{K}, g)$ as:

$$
\Delta T=\frac{1}{2}\left(\nabla_{k} \nabla_{\bar{k}}+\nabla_{\bar{k}} \nabla_{k}\right) T
$$

A Kähler metric $g$ defines a corresponding Riemannian metric $g_{R}$ on $(\mathcal{K}, g)$, defined via its real and imaginary parts, as follows. In local coordinates ${ }^{15}$ We can extend the covariant derivatives $\left(\nabla_{k}, \nabla_{\bar{k}}\right) \in(\mathcal{K}, g)$ to act naturally on any type of tensor field on $(\mathcal{K}, g)$; e.g., in case of covariant 2 -tensors $S_{i j}$ and $S_{\overline{i j}}$, we have:

$$
\begin{aligned}
& \nabla_{k} S_{\overline{l j}}=\partial_{k} S_{\overline{i j}}-\Gamma_{i k}^{p} S_{\overline{p j}}, \quad \nabla_{\bar{k}} S_{\bar{j}}=\partial_{\bar{k}} S_{\overline{i j}}-\Gamma_{\bar{j}}^{\bar{p}} S_{\bar{\pi}}, \\
& \nabla_{k} S_{i j}=\partial_{k} S_{i j}-\Gamma_{i k}^{p} S_{p j}-\Gamma_{j k}^{p} S_{i p}, \quad \nabla_{\bar{k}} S_{i j}=\partial_{\bar{k}} S_{i j} .
\end{aligned}
$$

Similarly, if $T$ is a mixed 3-tensor with components $T_{k}^{\bar{j}}$ then we define its two covariant derivatives $\nabla_{m} T_{k}^{\bar{j}}$ and $\nabla_{\bar{m}} T_{k}^{\bar{j}}$, as:

$$
\begin{gathered}
\nabla_{m} T_{k}^{\bar{j}}=\partial_{m} T_{k}^{\bar{j}}+\Gamma_{l m}^{i} T_{k}^{\bar{j}}-\Gamma_{k m}^{l} T_{l}^{\bar{j}}, \\
\nabla_{\bar{m}} T_{k}^{\bar{j}}=\partial_{\bar{m}} T_{k}^{\bar{j}}+\overline{\Gamma_{l m}^{j}} T_{k}^{\bar{i}} .
\end{gathered}
$$

${ }^{16}$ For example, the trace $\operatorname{Tr}(\cdot)$ of a real $(1,1)$-form $\alpha=\mathrm{i} \alpha_{i \bar{j}} d z^{i} \wedge d \overline{z^{j}} \in \mathcal{K}$ is defined by:

$$
\operatorname{Tr}_{\omega}(\alpha)=g^{\overline{j i}} \alpha_{i \bar{j}}=n \omega^{n-1} \wedge \alpha / \omega^{n} .
$$

Here, $\omega^{n}$ is the volume form on $\mathcal{K}$ (see below), such that for any smooth function $f \in \mathcal{K}$, its $L^{p}$-norm (with respect to a Kähler metric $g$ ) is given by: $\left(\int_{\mathcal{K}}|f|^{p} \omega^{n}\right)^{1 / p}$.

${ }^{17} \mathrm{~A}$ normal coordinate system for $g$ centered at a point $p \in \mathcal{K}$ is a holomorphic coordinate system that satisfies: $g_{i j}(p)=\delta_{i j}$ and $\partial_{k} g_{\overline{i j}}(p)=0$, which implies that the Christoffel symbols $\Gamma_{j k}^{i}$ of $g$ vanish at $p$. 
$\left\{z^{i}, \bar{z}^{i}\right\} \in \mathcal{K}$, we write $z^{i}=x^{i}+\mathrm{i} y^{i}$, so that $\partial_{z^{i}}=\frac{1}{2}\left(\partial_{x^{i}}-\mathrm{i} \partial_{y^{i}}\right)$ and $\partial_{\bar{z}^{i}}=\frac{1}{2}\left(\partial_{x^{i}}+\mathrm{i} \partial_{y^{i}}\right)$, which gives:

$$
g_{R}\left(\partial_{x^{i}}, \partial_{x^{j}}\right)=g_{R}\left(\partial_{y^{i}}, \partial_{y^{j}}\right)=2 \operatorname{Re}\left(g_{\overline{i j}}\right), \quad g_{R}\left(\partial_{x^{i}}, \partial_{y^{j}}\right)=2 \operatorname{Im}\left(g_{\overline{i j}}\right) .
$$

The Riemann curvature tensor $\mathrm{Rm}$ of the Kähler metric $g \in(\mathcal{K}, g)$ is very simply defined in two forms, mixed and covariant, respectively:

$$
R_{i k \bar{l}}^{m}=-\partial_{\bar{l}} \Gamma_{i k}^{m} \quad \text { and } \quad R_{\bar{i} k \bar{l}}=g_{m \bar{j}} R_{i k \bar{l}}^{m} .
$$

Using (9) and (10), we have locally (in an open chart $\left\{z^{i}, \bar{z}^{i}\right\} \in U \subset \mathcal{K}$; see [12]):

$$
R_{\bar{i} \hat{k} \bar{l}}=-\partial_{i} \partial_{\bar{j}} g_{k \bar{l}}+g^{\bar{q} p}\left(\partial_{i} g_{k \bar{q}}\right)\left(\partial_{\bar{j}} g_{p \bar{l}}\right) .
$$

The Riemann curvature tensor $R_{i \bar{i} k \bar{l}}$ on $(\mathcal{K}, g)$ has the following three symmetries: ${ }^{18}$

1) $\overline{R_{\bar{i} \hat{k} \bar{l}}}=R_{j \bar{j} \bar{k} \bar{k}}$ (complex-conjugate);

2) $R_{\bar{i} k \bar{l}}-R_{k \overline{k j} \bar{l}}-R_{i \bar{l} \bar{j} \bar{j}}$ (I Bianchi identity); and

3) $\nabla_{m} R_{i j k \bar{l}}=\nabla_{i} R_{m j k \bar{l}}$ (II Bianchi identity).

For any two nonzero vectorfields $(v, u)$ on $T \mathcal{K}$, we say that $(\mathcal{K}, g)$ has positive holomorphic bisectional curvature and positive holomorphic sectional curvature, respectively, if

$$
R_{\bar{j} \bar{k} l} \nu^{i} v^{\bar{j}} u^{k} u^{\bar{l}}>0 \text { and } R_{i \bar{j} k l} v^{i} v^{\bar{j}} v^{k} v^{\bar{l}}>0 .
$$

The trace of the Riemann curvature tensor $R_{i \bar{j} k \bar{l}}$ is the Ricci curvature tensor Rc, defined as:

$$
R_{\overline{i j}}=g^{\overline{l k}} R_{\bar{i} \bar{k} l}=g^{\overline{l k}} R_{k \overline{l i j}}=R_{k i \bar{j}}^{k},
$$

which locally (in an open chart $\left\{z^{i}, \bar{z}^{i}\right\} \in U \subset \mathcal{K}$; see [12] [14]) reads:

$R_{\overline{i j}}=-\partial_{i} \partial_{\bar{j}} \log \left[\operatorname{det}\left(g_{\overline{i j}}\right)\right]=-\partial \bar{\partial} \log [\operatorname{det}(g)]$.

Similarly, the trace of the Ricci curvature is the scalar curvature: $R=g^{\overline{j i}} R_{\overline{i j}}$.

A Kähler metric $g$ defines a pointwise norm $|\cdot|_{g}$ on any tensor field on $(\mathcal{K}, g)$; e.g., the squared norm of functions $f$ on $\mathcal{K}$ reads: $|\nabla f|^{2}=g^{i j} \partial_{i} f \partial_{\bar{j}} f$, and similarly for the vectorfields $(v, u) \in T \mathcal{K}$ and co-vectorfields $(\alpha, \beta) \in T^{*} \mathcal{K}$ we have: ${ }^{19}$

${ }^{18}$ While the symmetries 1 ) and 2) follow immediately from (9) and (11), to show 3 ) we need to compute at a point $p \in(\mathcal{K}, g)$ in normal coordinates for $g$.

$$
\nabla_{m} R_{\bar{j} \bar{k} \bar{l}}=-\partial_{m} \partial_{i} \partial_{\bar{j}} g_{k \bar{l}}=-\partial_{i} \partial_{m} \partial_{\bar{j}} g_{k \bar{l}}=\nabla_{i} R_{m \bar{j} k \bar{l}} .
$$

${ }^{19}$ The norm $|\cdot|_{g}$ extends to any tensor field on $\mathcal{K}$; e.g., if $T$ is a tensor with components $T_{k}^{\bar{j}}$, its squared norm is given by:

$$
|T|_{g}^{2}=g^{\bar{m} k} g_{\overline{i j}} g_{p \bar{q}} T_{k}^{i \bar{q}} \overline{T_{m}^{\bar{p}}}
$$

which in case of the Ricci tensor Rc and the Riemann tensor Rm become, respectively:

$$
|\mathrm{Rc}|^{2}=g^{i \bar{\ell}} g^{\bar{k}} R_{\overline{i j}} R_{k \bar{\ell}} \quad \text { and } \quad|\mathrm{Rm}|^{2}=g^{i \bar{q}} g^{p \bar{j}} g^{k \bar{s}} g^{r \bar{\ell}} R_{\overline{i j k} \bar{\ell}} R_{p \bar{q} \bar{r} \cdot} .
$$




$$
\begin{gathered}
|v|_{g}^{2}=g_{\overline{i j}} v^{i} \overline{v^{j}}, \quad|u|_{g}^{2}=g_{i \bar{j}} u^{\bar{j}} \overline{u^{\bar{i}}} \\
|\alpha|_{g}^{2}=g^{\overline{j i}} \alpha_{i} \overline{\alpha_{j}}, \quad|\beta|_{g}^{2}=g^{\overline{j i}} \beta_{\bar{j}} \overline{\beta_{\bar{i}}} .
\end{gathered}
$$

Associated to the Ricci curvature tensor Rc is the Ricci form, $\operatorname{Ric}(g) \equiv \operatorname{Ric}(\omega)$, a real closed $(1,1)$-form on $\mathcal{K}$, similar to the Kähler form $\omega$, given by:

$$
\operatorname{Ric}(\omega)=\mathrm{i} R_{\overline{i j}}(g) d z^{i} \wedge d z^{\bar{j}}=-\mathrm{i} \partial \bar{\partial} \log [\operatorname{det}(g)],
$$

which implies that $\operatorname{Ric}(\omega)$ is closed: $d \operatorname{Ric}(\omega)=0$.

The Riemann curvature tensor $R_{i j k \bar{l}}$ arises when commuting covariant derivatives $\left(\nabla_{k}, \nabla_{\bar{l}}\right) \in(\mathcal{K}, g)$. Using the standard commutator definition: $\left[\nabla_{k}, \nabla_{\bar{l}}\right]=\nabla_{k} \nabla_{\bar{l}}-\nabla_{\bar{l}} \nabla_{k}$, we have the following commutation formulae for the vectorfields $(v, u)$ on $T \mathcal{K}$ and co-vectorfields $(\alpha, \beta)$ on $T^{*} \mathcal{K}$ :

$$
\begin{aligned}
& {\left[\nabla_{k}, \nabla_{\bar{l}}\right] v^{m}=R_{i k \bar{l}}^{m} v^{i}, \quad\left[\nabla_{k}, \nabla_{\bar{l}}\right] u^{\bar{m}}=-R_{\bar{j} k \bar{l}}^{\bar{m}} u^{\bar{j}},} \\
& {\left[\nabla_{k}, \nabla_{\bar{l}}\right] \alpha_{i}=-R_{i k \bar{l}}^{m} \alpha_{m}, \quad\left[\nabla_{k}, \nabla_{\bar{l}}\right] \beta_{\bar{j}}=R_{\bar{j} k \bar{m}}^{\bar{m}} \beta_{\bar{m}},}
\end{aligned}
$$

which can naturally be extended to tensors of any type on $\mathcal{K}$. Also, when acting on any tensor, the covariant derivatives commute as: $\left[\nabla_{i}, \nabla_{j}\right]=\left[\nabla_{\bar{i}}, \nabla_{\bar{j}}\right]=0$.

Now we come to the essential notion of cohomology of a Kähler manifold $(\mathcal{K}, \omega)$, which is defined using the formalism of $(\partial, \bar{\partial})$-operators. Recall that de Rham's cohomology group $H_{d}^{2}(\mathcal{K}, \mathbb{R})$, based on the exterior derivative $d=\partial+\bar{\partial},{ }^{20}$ considers a symplectic 2 -form $\alpha \in \mathcal{K}$ which is globally closed: $d \alpha=0$ and locally exact: $\alpha=d \eta$, for some canonical 1-form $\eta$ (Poincaré lemma). Then the group $H_{d}^{2}(\mathcal{K}, \mathbb{R})$ is defined as the quotient space: ${ }^{21}$

$$
H_{d}^{2}(\mathcal{K}, \mathbb{R})=\frac{\{d \text {-closed real } 2 \text {-forms }\}}{\{d \text {-exact real } 2 \text {-forms }\}} .
$$

Similarly, a $(1,1)$-form $\alpha \in(\mathcal{K}, \omega)$ is called $\bar{\partial}$-closed if $\bar{\partial} \alpha=0$ and $\bar{\partial}$ -exact if $\alpha=\bar{\partial} \eta$ for some $(0,1)$-form $\eta .^{22}$ Therefore, a complexification of de Rham's group $H_{d}^{2}(\mathcal{K}, \mathbb{R})$ gives the Dolbeault cohomology group $H_{\bar{\partial}}^{1,1}(\mathcal{K}, \mathbb{R})$, defined as the quotient space: ${ }^{23}$

\footnotetext{
${ }^{20}$ The closure relation between these three derivative operators reads:

$$
d(i \partial \bar{\partial})=\mathrm{i}(\partial+\bar{\partial}) \partial \bar{\partial}=\mathrm{i}\left(\partial^{2} \bar{\partial}-\partial \bar{\partial}^{2}\right)=0 .
$$
}

${ }^{21}$ For general $p$-forms, the de Rham cohomology group $H_{d}^{p}(\mathcal{K}, \mathbb{R})$ is defined as the kernel over the image of the exterior derivative $d$ :

$$
H_{d}^{p}(\mathcal{K}, \mathbb{R})=\frac{\operatorname{Ker}\left[d: \Omega^{p}(\mathcal{K}) \rightarrow \Omega^{p+1}(\mathcal{K})\right]}{\operatorname{Im}\left[d: \Omega^{p-1}(\mathcal{K}) \rightarrow \Omega^{p}(\mathcal{K})\right]} .
$$

${ }^{22}$ This is the $\bar{\partial}$-Poincaré lemma for complex manifolds, which says that a $\bar{\partial}$-closed $(0,1)$--form is locally $\bar{\partial}$-exact.

${ }^{23}$ For general $(p, q)$-forms, the Dolbeault cohomology group $H_{\bar{\partial}}^{p, q}(\mathcal{K}, \mathbb{R})$ is defined as the kernel over the image of the $\bar{\partial}$-operator:

$$
H_{\bar{\partial}}^{p, q}(\mathcal{K}, \mathbb{R})=\frac{\operatorname{Ker}\left[\bar{\partial}: \Omega^{p, q}(\mathcal{K}) \rightarrow \Omega^{p, q+1}(\mathcal{K})\right]}{\operatorname{Im}\left[\bar{\partial}: \Omega^{p, q-1}(\mathcal{K}) \rightarrow \Omega^{p, q}(\mathcal{K})\right]} .
$$




$$
H_{\bar{\partial}}^{1,1}(\mathcal{K}, \mathbb{R})=\frac{\{\bar{\partial} \text {-closed real }(1,1) \text {-forms }\}}{\{\bar{\partial} \text {-exact real }(1,1) \text {-forms }\}} .
$$

A Kähler form $\omega$ on $(\mathcal{K}, \omega)$ defines a nonzero element $[\omega]$ of $H_{\bar{\partial}}^{1,1}(\mathcal{K}, \mathbb{R})$. If a cohomology class $\alpha \in H_{\bar{\partial}}^{1,1}(\mathcal{K}, \mathbb{R})$ can be written as: $\alpha=[\omega]$, for some Kähler form $\omega$, then we say that $\alpha$ is a Kähler class and write $\alpha>0 .{ }^{24}$

As already mentioned, the famous $\partial \bar{\partial}$-lemma (which is the holomorphic version of the classic Poincaré lemma that follows from Hodge theory) is the fundamental result of Kähler geometry. Let $(\mathcal{K}, \omega)$ be a compact Kähler manifold and suppose that $0=[\alpha] \in H_{\bar{\partial}}^{1,1}(\mathcal{K}, \mathbb{R})$ for a real smooth $\bar{\partial}$-closed $(1,1)$-form $\alpha$ on $(\mathcal{K}, \omega)$. Then there exists a real-valued smooth function $\varphi \in(\mathcal{K}, \omega)$, called the Kähler potential, such that the form $\alpha$ is uniquely determined (up to the addition of a constant) as: $:^{25}$

$$
\alpha=\mathrm{i} \partial_{i} \partial_{\bar{j}} \varphi=\mathrm{i} \partial \bar{\partial} \varphi>0 .
$$

In other words, a real $(1,1)$-form $\alpha$ is $\bar{\partial}$-exact iff it is $\partial \bar{\partial}$-exact. ${ }^{26}$ An immediate consequence is that if $\omega$ and $\omega_{\varphi}$ are two Kähler forms in the same Kähler class $[\omega] \in H_{\vec{\partial}}^{1,1}(\mathcal{K}, \mathbb{R})$, then we have:

$$
\omega_{\varphi}=\omega+\mathrm{i} \partial \bar{\partial} \varphi>0,
$$

for some smooth Kähler potential $\varphi$ (which is uniquely determined up to a constant). In other words, two Kähler metrics $g_{\overline{i j}}$ and $\tilde{g}_{\overline{i j}}$ on $(\mathcal{K}, g)$ belong to the same Kähler class iff

${ }^{24}$ In other words, a Kähler form $\omega$ on $\mathcal{K}$ defines a nonzero element $[\omega] \in H_{\bar{\partial}}^{1,1}(\mathcal{K}, \mathbb{R})$. If a cohomology class $\alpha \in H_{\bar{\partial}}^{1,1}(\mathcal{K}, \mathbb{R})$ can be written as $\alpha=[\omega]$ for some Kähler form $\omega$ on $\mathcal{K}$ then we say that $\alpha$ is a Kähler class (and write $\alpha>0$ ). Therefore, the Kähler class of $\omega$ is its cohomology class $[\omega] \in H_{\bar{\partial}}^{1,1}(\mathcal{K}, \mathbb{R})$. Alternatively, in terms of $H_{d}^{2}(\mathcal{K}, \mathbb{R})$, the Kähler class of $\omega$ is its cohomology class $[\omega] \in H_{d}^{2}(\mathcal{K}, \mathbb{R})$. Usually, all this is simply written: the Kähler class of $\omega$ is the cohomology class $[\omega] \in H^{2}(\mathcal{K}, \mathbb{R})$ represented by $\omega$.

${ }^{25}$ The simplest example of the Kähler potential is the case of the simplest Kähler $n$-manifold $\mathbb{C}^{n}$-the complex Euclid $n$-space, in which $\varphi \in \mathbb{C}^{n}$ is given by:

$$
\varphi\left(z^{i}, \bar{z}^{i}\right)=\frac{1}{2} \sum_{i}^{n} z^{i} \bar{z}^{i} .
$$

Another standard example is the following entropic Kähler potential (see [16]) with its Taylor expansion:

$$
\begin{aligned}
& \varphi\left(z^{i}, \bar{z}^{i}\right)=-\log \left(1-\sum_{i}^{n} z^{i} \bar{z}^{i}+\frac{1}{4}\left|\sum_{i}^{n}\left(z^{i}\right)^{2}\right|^{2}\right) \\
& \approx \sum_{i}^{n} z^{i} \bar{z}^{i}+\frac{1}{4} \sum_{i}^{n}\left(z^{i}\right)^{2}\left(\bar{z}^{i}\right)^{2}+\sum_{i<j}^{n}\left[z^{i} \bar{z}^{i} z^{j} \bar{z}^{j}-\frac{1}{4}\left(z^{i}\right)^{2}\left(\bar{z}^{j}\right)^{2}-\frac{1}{4}\left(z^{j}\right)^{2}\left(\bar{z}^{i}\right)^{2}\right] .
\end{aligned}
$$

${ }^{26}$ The $\partial \bar{\partial}$-lemma is an extension of the two Poincaré lemmas (for $d$ and $\bar{\partial}$ ). Basically, the $\partial \bar{\partial}$ -lemma says that a closed $(1,1)$-form $\alpha$ locally arises as $\mathrm{i} \partial \bar{\partial} \varphi$ for some smooth function $\varphi$, called the Kähler potential. This is similar to the standard Poincaré lemma, which says that closed exterior forms, $d \alpha=0$, are locally exact, $\alpha=d \varphi$, where "exactness" means $d$ (something), which is true on all smooth manifolds. The $\bar{\partial}$-Poincaré lemma, in which "exactness" means $\bar{\partial}$ (something), is true on all complex manifolds. The $\partial \bar{\partial}$-lemma, in which "exactness" means $\partial \bar{\partial}$ (something), is true only on Kähler manifolds. Therefore, Kähler manifolds are the most important of all: $\partial \bar{\partial}$-lemma $\Rightarrow \bar{\partial}$-Poincaré lemma $\Rightarrow$ Poincaré lemma. 


$$
g_{\bar{j}}=\tilde{g}_{\bar{j}}+\partial_{i} \partial_{\bar{j}} \varphi .
$$

The volume form: $\omega^{n}\left(=n ! \omega^{[n]}\right)$ and the standard volume $\operatorname{Vol}_{\omega}$ on $(\mathcal{K}, \omega)$ are given, respectively, by:

$$
\omega^{[n]}=\mathrm{i}^{n} \operatorname{det}(g) d z^{1} \wedge d z^{\overline{1}} \wedge \cdots \wedge d z^{n} \wedge d z^{\bar{n}}, \quad \operatorname{Vol}_{\omega}=\int_{\mathcal{K}} \omega^{[n]},
$$

so that: $\partial \bar{\partial} \log \left(\omega^{n}\right)=\partial \bar{\partial} \log [\operatorname{det}(g)]$. By the universal Stokes theorem, if $\omega$ and $\tilde{\omega}$ are two Kähler forms in the same Kähler class $[\omega] \in H_{\vec{\partial}}^{1,1}(\mathcal{K}, \mathbb{R})$ then: $\operatorname{Vol}_{\omega}=\operatorname{Vol}_{\tilde{\omega}}$. The total scalar curvature is determined by the Ricci form $\operatorname{Ric}(\omega)$ as [14]:

$$
\int_{\mathcal{K}} R \omega^{[n]}=\int_{\mathcal{K}} \operatorname{Ric}(\omega) \wedge \omega^{[n-1]},
$$

and it depends only on the Kähler class $[\omega] \in H_{\vec{\partial}}^{1,1}(\mathcal{K}, \mathbb{R})$ and the first Chern class, $c_{1}(\mathcal{K})$, defined as the cohomology class of the Ricci form: $[\operatorname{Ric}(\omega)] \in H_{\bar{\partial}}^{1,1}(\mathcal{K}, \mathbb{R})$.

The space $\mathcal{K}_{[\omega]}$ of Kähler forms $\omega$ on $(\mathcal{K}, \omega)$ with the same Kähler class $[\omega] \in H_{\vec{\partial}}^{1,1}(\mathcal{K}, \mathbb{R})$ is given by:

$$
\mathcal{K}_{[\omega]}=\left\{[\omega] \in H^{2}(\mathcal{K}, \mathbb{R}) \mid \omega+\mathrm{i} \partial \bar{\partial} \varphi>0\right\},
$$

and the associated functional space $\mathcal{H}$ of Kähler potentials $\varphi \in(\mathcal{K}, \omega)$ in the class $[\omega]$ is given by $[15]$ :

$$
\mathcal{H}=\left\{\varphi \in C^{\infty}(\mathcal{K}, \mathbb{R}) \mid \omega_{\varphi}=\omega+\mathrm{i} \partial \bar{\partial} \varphi>0\right\},
$$

for which the geodesic equation (w.r.t. $\omega_{\varphi}$ ) reads: ${ }^{27}$

$$
\ddot{\varphi}-|\nabla \dot{\varphi}|^{2}=0, \quad \varphi(0)=\varphi_{0} .
$$

Based on the sign of their first Chern class $c_{1}(\mathcal{K})=[\operatorname{Ric}(\omega)] \in H_{\vec{\partial}}^{1,1}(\mathcal{K}, \mathbb{R})$, all compact Kähler manifolds $(\mathcal{K}, \omega)$ can be classified into the following three categories:

- $(\mathcal{K}, \omega)$ with positive first Chern class, $c_{1}(\mathcal{K})>0$, is called the Fano manifold in which $[\operatorname{Ric}(\omega)]=\pi c_{1}(\mathcal{K})$. It admits Kähler-Ricci solitons, metrics for which:

$$
\operatorname{Ric}(\omega)-\omega=\mathfrak{L}_{v} \omega,
$$

where $\mathfrak{L}_{v}$ is the Lie derivative along a holomorphic vector field $v=v^{a} \in \mathcal{K}$.

- $(\mathcal{K}, \omega)$ with vanishing first Chern class, $c_{1}(\mathcal{K})=0$, is called the Calabi-Yau manifold, the basic geometric object in string theory.

- $(\mathcal{K}, \omega)$ with negative first Chern class, $c_{1}(\mathcal{K})<0$, is called the Kähler-Einstein manifold, which admits the Kähler-Einstein metric $g$ defined by:

$$
R_{\overline{i j}}=\lambda g_{\overline{i j}}(\text { or } \operatorname{Ric}(\omega)=\lambda \omega) \text {, with } \lambda=\frac{2 \pi}{\operatorname{Vol}_{\omega}} \int_{\mathcal{K}} c_{1}(\mathcal{K}) \wedge \omega^{n-1} .
$$

In addition, if $\operatorname{Ric}(g)=0$ on $(\mathcal{K}, g)$ then $g$ is a Ricci-flat metric. In that ${ }^{27}$ The geodesic equation is equivalent [11] to the homogeneous complex Monge-Amperè equation (see below):

$$
\left(\ddot{\varphi}-|\nabla \dot{\varphi}|^{2}\right) \frac{\omega_{\varphi}^{n}}{\omega^{n}}=0 .
$$


case, according to the Calabi conjecture (see [16]) proven by S.-T. Yau [17], the first Chern class must also vanish: $c_{1}(\mathcal{K})=0 .{ }^{28}$

\subsection{Kähler-Ricci Flow}

Now we are ready to introduce our main actor, the Kähler-Ricci flow (see [6] [18] and the references therein) on a Kähler manifold $(\mathcal{K}, g)$. For this, we firstly recall that the real-valued Ricci flow on a Riemannian manifold $M$ (introduced by R. Hamilton [19] [20] [21] and subsequently used by G. Perelman to prove the 100-year old Poincaré Conjecture, the only solved Clay Research's Millennium Prize problem), is governed by the nonlinear evolution equation of the Riemannian metric (in real components $i, j=1, \cdots, n$ ):

$$
\partial_{t} g_{i j}(t)=-2 R_{i j}(t), \quad g_{i j}(0)=g_{0}
$$

which in local harmonic coordinates on $M$ can be rewritten in terms of the Laplace-Beltrami operator $\Delta$ as:

$$
\partial_{t} g_{i j}(t)=\Delta g_{i j}+Q_{i j}\left(g_{i j}, \partial g_{i j}\right), \quad g_{i j}(0)=g_{0},
$$

where the tensor function $Q_{i j}\left(g_{i j}, \partial g_{i j}\right)$ is quadratic in $g_{i j}$ and its first order partial derivatives, $\partial g_{i j}$. Later, in [18], Equations (14)-(15) were proposed as a general model for a wide range of (real) nonlinear reaction-diffusion systems.

The Ricci flow (14) has a unique solution, called a gradient Ricci soliton, only in case of Einstein manifolds, such that $R_{a b}=\lambda g_{a b}$, which can be shrinking if $\lambda>0$, steadyif $\lambda=0$ and expanding if $\lambda<0 .^{29}$

The complexification of the real Ricci flow (14), from a Riemannian manifold $\left(M, g_{i j}\right)$ of real dimension $n$ to the Kähler manifold $\left(\mathcal{K}, g_{\overline{i j}}\right)$ of complex dimension $n$, is called the Kähler-Ricci flow (KRF), given by (see, e.g. [14] and [12]):

$$
\partial_{t} \omega=-\operatorname{Ric}(\omega), \quad \omega(0)=\omega_{0},
$$

${ }^{28}$ The Calabi-Yau theorem says that if $(\mathcal{K}, \omega)$ is a compact Kähler manifold and $\alpha$ is a real $(1,1)$-form representing $c_{1}(\mathcal{K})$ then there exists a unique Kähler metric $g$ on $\mathcal{K}$ with $[g]=[\omega]$, such that $\operatorname{Ric}(g)=2 \pi \alpha$.

${ }^{29} \mathrm{~A}$ Riemannian metric $g$ is called Einstein metric if the Ricci curvature of the metric is some multiple of the metric, that is, the Ricci tensor $R_{a b}(g)$ is defined as $R_{a b}=\lambda g_{a b}$ for some constant $\lambda$. A smooth manifold $(M, g)$ with an Einstein metric $g$ is called an Einstein manifold.

If the initial metric $g(0)$ is Ricci flat $\left(R_{a b}=0\right)$, then $\partial_{t} g_{a b}=0$, so $g_{a b}=$ const under (12). Hence any Ricci flat metric is a stationary solution of the Ricci flow (12). If the initial metric is an Einstein metric of positive scalar curvature $(R>0)$, then the metric will shrink homothetically under the Ricci flow (12) by a time-dependent factor, and if the initial metric is an Einstein metric of negative scalar curvature $(R<0)$, the metric will expand homothetically for all times.

Therefore, the following simple and conformal solution of the Ricci flow (12) uniquely exists on Einstein $n$-manifolds (with $R_{a b}=\lambda g_{a b}$ ):

$$
g_{a b}(x, t)=\rho^{2}(t) g_{a b}(x, 0), \quad \text { or simply: } g(t)=\rho^{2}(t) g(0),
$$

where in case of the positive scalar curvature ( $R>0)$, the function $\rho(t)$ satisfies the ODE:

$$
\dot{\rho}=-\lambda / p, \quad \text { with the shrinking solution: } \rho^{2}(t)=1-2 \lambda t,
$$

and in case of the negative scalar curvature $(R<0)$, the function $\rho(t)$ satisfies the ODE:

$$
\dot{\rho}=\lambda / p, \quad \text { with the expanding solution: } \rho^{2}(t)=1+2 \lambda t \text {. }
$$


with the extended form: ${ }^{30}$

$$
\partial_{t} \omega=-\operatorname{Ric}(\omega)-\lambda \omega, \quad \omega(0)=\omega_{0},
$$

where the real constant $\lambda$ is either 0 or 1 . The case $\lambda=1$ gives a rescaling of (16) called the normalized KRF.

In particular, a Fano $n$-manifold $(\mathcal{K}, g)$ with positive first Chern class, $c_{1}(\mathcal{K})>0$, in which $[\operatorname{Ric}(\omega)]=\pi c_{1}(\mathcal{K})$, admits the normalized KRF (see [14] [22]) with the time-dependent Ricci form (12) $\operatorname{Ric}(\omega)=\operatorname{Ric}[\omega(t)]$, given by: ${ }^{31}$

$$
\partial_{t} g_{i j}(t)=g_{i j}(t)-\operatorname{Ric}[\omega(t)], \quad g_{i \bar{j}}(0)=g_{0},
$$

which is, starting from some smooth initial Kähler metric tensor $g_{0}$ given locally (in an open chart $U \subset \mathcal{K}$ ) by:

$$
\partial_{t} g_{\overline{i j}}(t)=g_{\overline{i j}}(t)-R_{\overline{i j}}(t), \quad g_{\overline{i j}}(0)=g_{0} .
$$

The normalized KRF (18) preserves the Kähler class $[\omega]$. It has a global solution $g(t) \equiv \omega(t)$ when $g_{0}=g_{\overline{i j}}(0)$ has $[\omega]=2 \pi c_{1}(\mathcal{K})$ as its Kähler class [which is written as $g_{0} \in 2 \pi c_{1}(\mathcal{K})$ ].

In terms of time-dependent Kähler potentials $\varphi=\varphi(t)$, the KRF (18) can be expressed as:

$$
\partial_{t} \varphi(t)=\varphi(t)+\log \left(\omega_{\varphi}^{n} / \omega^{n}\right)-g(t), \quad \varphi(0)=\varphi_{0},
$$

where the time-dependent Kähler metric $g=g(t)$ is defined by:

$$
\mathrm{i} \partial \bar{\partial} g(t)=\operatorname{Ric}[\omega(t)]-\omega(t) \text { and } \int_{\mathcal{K}}\left(\mathrm{e}^{g(t)}-1\right) \omega^{n}=0 .
$$

The corresponding evolutions of the Ricci curvature $R_{\overline{i j}}=R_{\overline{i j}}(t)$ and the scalar curvature $R=R(t)$ on $(\mathcal{K}, g)$ are governed, respectively by:

$$
\partial_{t} R_{\overline{i j}}=\Delta R_{\overline{i j}}+R_{\overline{i j p} \bar{q}} R_{q \bar{p}}-R_{\overline{i p}} R_{\overline{p j}}, \quad \partial_{t} R=\Delta R+R_{\overline{i j}} R_{\bar{j} \bar{i}}-R,
$$

starting from some smooth initial Ricci and scalar curvatures, $R_{\overline{i j}}(0)$ and $R(0)$.

The evolution of the scalar curvature $R$ can be also expressed in terms of the Ricci form as:

$$
\partial_{t} R=\Delta R+|\operatorname{Ric}(\omega)|^{2}+\lambda R, \quad R(0)=R_{0},
$$

and it has a lower bound (determined by the real constant: $C=-\inf _{\mathcal{K}} R(0)-\lambda n$; see [12]):

$$
R(t) \geq-\lambda n-C \mathrm{e}^{-\lambda t} .
$$

The corresponding time evolution of the trace of the metric, $\operatorname{Tr}(\omega)=\operatorname{Tr}_{\omega}(g)$, ${ }^{30}$ Similar to the KRF is the Calabi flow determined by the scalar curvature: $\partial_{t} \omega=\mathrm{i} \partial \bar{\partial} R$.

${ }^{31}$ The KRF (18) preserves the Kähler class $[\omega]$. It has a global solution $g(t) \equiv \omega(t)$ when $g_{0}=g_{i j}(0)$ has $[\omega]=2 \pi c_{1}(\mathcal{K})$ as its Kähler class, which is written as $g_{0} \in 2 \pi c_{1}(\mathcal{K})$. In particular by the $\partial \bar{\partial}$-Lemma, there exists a family of real-valued functions $u(t)$, called Ricci potentials of the metric $g(t)$, which are special Kähler potentials. They are determined by the volume $V_{R}$ of the $\operatorname{KRF}(18)$ :

$$
g_{\overline{i j}}-R_{i \bar{j}}=\partial_{i} \partial_{\bar{j}} u, \quad \frac{1}{V_{R}} \int_{\mathcal{K}} \mathrm{e}^{-u(t)} \mathrm{d} v_{g}=1, \quad V_{R}=\int_{\mathcal{K}} \mathrm{d} v_{g} .
$$


computed in normal coordinates for the metric $g$ (see [12]), and its lower bound, are given respectively by:

$$
\begin{gathered}
\partial_{t} \operatorname{Tr}(\omega)=-g^{\overline{j i}} R_{\overline{i j}}-\lambda \operatorname{Tr}(\omega),\left.\quad \operatorname{Tr}(\omega)\right|_{\omega_{0}}=\operatorname{Tr}\left(\omega_{0}\right), \\
\left(\partial_{t}-\Delta\right) \log [\operatorname{Tr}(\omega)] \leq C \operatorname{Tr}(\omega)-\lambda
\end{gathered}
$$

In general, the existence of the KRF in a time interval $t \in\left[0, t_{1}\right)$ can be established as follows: if $\omega(t)$ is a solution of the KRF:

$$
\partial_{t} \omega(t)=-\operatorname{Ric}[\omega(t)], \quad \omega(0)=\omega_{0},
$$

then the corresponding cohomology class $[\omega(t)]$ evolves on $(\mathcal{K}, g)$ according to the following ODE:

$$
\begin{aligned}
& \partial_{t}[\omega(t)]=-c_{1}(\mathcal{K}), \quad[\omega(0)]=\left[\omega_{0}\right], \quad \text { with the solution: } \\
& {[\omega(t)]=\left[\omega_{0}\right]-t c_{1}(\mathcal{K})=[\mathrm{i} \partial \bar{\partial} \varphi(0)]-t c_{1}(\mathcal{K}), \quad \text { for }\left[t \in\left[0, t_{1}\right)\right] .}
\end{aligned}
$$

So, the KRF (19) exists for $t \in\left[0, t_{1}\right)$ iff $\left[\omega_{0}\right]-t c_{1}(\mathcal{K})>0$ (see [12] [14]).

In particular, the extensions of the Kähler-Einstein (KE) metric:

$$
R_{\overline{i j}}=\lambda g_{\overline{i j}} \Leftrightarrow \operatorname{Ric}(\omega)=\lambda \omega,
$$

are the Kähler-Ricci (KR) solitons: a time-dependent Kähler metric $g(t)=g_{\overline{i j}}(t)$ is called a gradient KR soliton if there exists a real smooth Kähler potential $\varphi$ on $(\mathcal{K}, g)$ such that:

$$
R_{i \bar{j}}=\lambda g_{\overline{i j}}-\partial_{i} \partial_{\bar{j}} \varphi \text { and } \nabla_{i} \nabla_{j} \varphi=0 \Leftrightarrow \nabla \varphi=\left(g^{\overline{i j}} \partial_{\bar{j}} \varphi\right) \partial_{i} .
$$

Similar to the real Ricci flow case, this soliton is called shrinking if $\lambda>0$, steady if $\lambda=0$, and expanding if $\lambda<0$, and the gradient vectorfield $\nabla \varphi$ is holomorphic. If the Kähler manifold $(\mathcal{K}, g)$ admits a KE metric (or, a KR soliton) $g$ then the first Chern class $c_{1}(\mathcal{K})$ is necessarily definite: $\pi c_{1}(\mathcal{K})=\lambda\left[\omega_{g}\right] .^{32}$

At the end of this section, we remark that it was shown by [17] [23] that the KRF (19) can be rewritten as the (parabolic, complex) Monge-Ampère equation. ${ }^{33}$

${ }^{32}$ For any Kähler metric $g \in 2 \pi c_{1}(\mathcal{K})$ with scalar curvature $R$ and for any smooth function $u$ on $\mathcal{K}$, the Kähler-Perelman $(\mathrm{KP})$ entropy $\mathcal{E} \equiv \mathcal{E}(g)$ is defined by:

${ }^{33} \mathrm{On} \mathbb{C}^{n}$ the complex Monge-Ampere operator for the smooth potential $\varphi$ is defined as the determinant of the complex Hessian:

$$
\operatorname{det}\left(\partial_{i} \partial_{\bar{j}} \varphi\right) \equiv \operatorname{det}\left(\frac{\partial^{2} \varphi}{\partial z^{i} \partial \bar{z}^{j}}\right), \quad \text { with }\left(\partial_{i} \partial_{\bar{j}} \varphi\right) \geq 0 .
$$

However, on a compact Kähler manifold $(\mathcal{K}, g)$, the condition $\left(\partial_{i} \partial_{\bar{j}} \varphi\right) \geq 0$ would imply that $\varphi$ is constant, so the Hessian $\left(\partial_{i} \partial_{\bar{j}} \varphi\right)$ is naturally replaced by $\left(g_{\overline{i j}}+\partial_{i} \partial_{\bar{j}} \varphi\right)$, to define the complex Monge-Ampère operator on $(\mathcal{K}, g)$ as:

$$
\operatorname{det}\left(g_{\bar{i}}+\partial_{i} \partial_{\bar{j}} \varphi\right) \equiv \operatorname{det}\left(g_{\overline{i j}}+\frac{\partial^{2} \varphi}{\partial z^{i} \partial \bar{z}^{j}}\right), \quad \text { with }\left(\partial_{i} \partial_{\bar{j}} \varphi\right) \geq 0 .
$$

Using this operator, the complex Monge-Ampère equation is defined as:

$$
\partial_{t} \varphi=\log \frac{\operatorname{det}\left(g_{\overline{i j}}+\partial_{i} \partial_{\bar{j}} \varphi\right)}{\operatorname{det}(g)}=\log \left[\frac{\left(\omega_{\varphi}+\mathrm{i} \partial \bar{\partial} \varphi\right)^{n}}{\omega^{n}}\right],
$$

since: $\partial \bar{\partial} \log \left(\omega^{n}\right)=\partial \bar{\partial} \log [\operatorname{det}(g)]$. 


$$
\partial_{t} \varphi=\log \left[\left(\omega_{\varphi}+\mathrm{i} \partial \bar{\partial} \varphi\right)^{n} / \omega^{n}\right], \omega_{0}+\mathrm{i} \partial \bar{\partial} \varphi>0, \varphi(0)=\varphi_{0},
$$

since we have (see [12]):

$$
\partial_{t} \omega(t)=\partial_{t} \omega_{\varphi}+\mathrm{i} \partial \bar{\partial}\left(\partial_{t} \varphi\right)=\partial_{t}\left(\omega_{\varphi}+\mathrm{i} \partial \bar{\partial} \varphi\right)=-\operatorname{Ric}[\omega(t)] .
$$

Similarly, the normalized KRF (18) with $\lambda=1$, that is:

$$
\partial_{t} \omega(t)=-\operatorname{Ric}[\omega(t)]-\omega(t), \omega(0)=\omega_{0},
$$

can be rewritten as a normalized (parabolic, complex) Monge-Ampère equation:

$$
\partial_{t} \varphi=\log \left[\left(\omega_{0}+\mathrm{i} \partial \bar{\partial} \varphi\right)^{n} / \omega^{n}\right]-\varphi, \omega_{0}+\mathrm{i} \partial \bar{\partial} \varphi>0, \varphi(0)=\varphi_{0} .
$$

For more technical details on the Kähler-Ricci flow, see e.g., [12] [14] and the references therein. 